Myrtia, $\mathrm{n}^{\circ} 36$ (2021), 9-28

La parodo dell'Eracle di Euripide:

tradizione critica della colometria e del testo

[The parodos of Euripides' Heracles:

tradition and criticism of colometric layout and text]

\title{
Paolo Santé*
}

Liceo 'C. Salutati', Montecatini Terme (PT) - Italia

\begin{abstract}
Resumen: $\quad$ Analizo el texto y la colometría de los mss. Laurentianus plut. 32,2 (L) y Laurentianus conv. soppr. $172+$ Palatinus gr. 287 (P) para el canto de entrada del coro de Heracles. Muchos lugares han sido discutidos y corregidos por un autorizado grupo de filólogos. Los discutiré nuevamente, proponiendo una defensa del texto antiguo y la colometría donde creo haber encontrado argumentos adecuados. Las ediciones modernas modifican la colometría en varios lugares, anulando la mezcla de cola yámbicos y trocaicos, que en cambio tiene su propia ratio. La presencia de los '` $\alpha \mu \beta o \iota$ podría remontarse al género ialemos, una forma de lamento fúnebre, y la

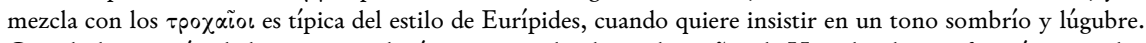
Cuando la atención de los ancianos de sí mismos se desplaza a los niños de Heracles, la versificación se vuelve

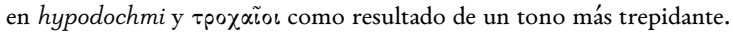

Abstract: $\quad$ This work analyzes text and colometry of mss. Laurentianus plut. 32,2 (L) and Laurentianus conv. soppr. $172+$ Palatinus gr. 287 (P) for the chorus' entrance song of Heracles. Many places have been discussed and corrected by an authoritative group of philologists. I will re-discuss them, proposing a defense of the ancient text and colometry, where I believe I have found suitable arguments. The modern editions modify the colometry in several places, cancelling the mixture of iambic and trochaic cola, which instead has its own ratio. The presence of ' $\alpha \mu \beta$ o c could be traced back to ialemos genus, a form of funeral lament, and the mixture with $\tau p \circ \chi \alpha \tilde{i} о \mathrm{~s}$ is typical of Euripide's style, when he wants to insist on a sombre and mournful tone. When the attention of the elderly people from themselves shifts to Heracles' children, the versification turns into

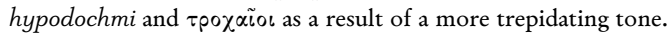

Palabras clave: crítica textual; colometría antigua; Eurípides; Heracles.

Keywords: $\quad$ textual criticism; ancient colometry; Euripides; Heracles.

$\begin{array}{lll}\text { Recepción: } & 17 / 12 / 2020 & \text { Aceptación: 31/01/2021 }\end{array}$

\section{Introduzione: il coro-cigno cantore di lamenti funebri}

Nel Prologo dell'Eracle a parlare è il vecchio Anfitrione, padre 'putativo' dell'eroe Eracle, nato da sua moglie Alcmena unitasi con Zeus (vv. 1-59). Accanto a lui compare Megara, moglie di Eracle, che prende la parola (vv. 60-86) per poi dialogare con Anfitrione (vv. 87-106). Accanto a Megara ci sono i tre figli piccoli, personaggi muti. ${ }^{1}$ Sulla scena c'è dunque l'intera famiglia di Eracle, non più tornato dopo essere sceso nell'Ade per la sua ultima fatica, la cattura del cane Cerbero. ${ }^{2} \mathrm{Di}$

\footnotetext{
* Dirección para correspondencia: via Provinciale Lucchese 157/A 51010, Santa Lucia di Uzzano (PT), Italia. Correo electrónico: paolo.sante@posta.istruzione.it. Ringrazio gli anonimi revisori per le osservazioni. Sono grato al prof. Pietro Giannini per avere letto e migliorato una prima versione di questo scritto.

1 Per il numero tre, $c f$. i vv. 474, 995 e 1023. Le varianti su numero e nome di questi ragazzini, figli di Eracle e Megara, sono numerose: $c f$. schol. ad Pi. I. 4, 104, p. 237, 16-24 e p. 238, 1-10 Drachmann. Lo scoliasta sembra ricordare una tragedia perduta (E. fr. 1016 Kannicht) perché nell'Eracle non si citano i loro nomi, dunque neppure Aristodemos, che sarebbe stato aggiunto da Euripide a Therimachos e Deikoon. Tuttavia potrebbe anche essere accaduto, come riassume R. Kannicht, $\operatorname{Tr} G F$ V 1-2, p. 197 che «e memoria grammatica vel mythographica ad illos

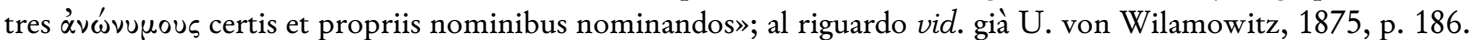

${ }^{2}$ Eracle ha lasciato a Tebe figli e moglie per purificare il padre Anfitrione e consentirgli di tornare ad Argo (vv. 13-17). Anfitrione, infatti, si era macchiato dell'assassinio del suocero Elettrione. Secondo la versione di Ps. Apollod. II, 4, 6, l'uccisione di Elettrione fu fortuita: mentre stava recuperando il bestiame, una delle giovenche si imbizzarrì e Anfitrione le scagliò contro un grosso bastone che, rimbalzando contro le corna dell'animale, colpì al capo Elettrione e lo uccise: cf. anche Tz. schol. ad Lyc. Alex. 932. Nella versione di Ps. Hes. Sc. 11-12 e 81-82,
} 
fronte alla facciata del palazzo di Eracle, i suoi familiari hanno trovato rifugio presso l'altare di Zeus Salvatore ${ }^{3}$ che, un tempo, proprio Eracle aveva innalzato dopo la vittoria sui Mini (vv. 49-50). Il loro intento è sottrarsi all'usurpatore Lico che, dopo avere ucciso il re Creonte, ha assunto il potere a Tebe e li minaccia di morte, timoroso di una futura vendetta per le sue malefatte. ${ }^{4}$ Le loro speranze di salvezza appaiono fragili, per una condizione di estrema debolezza: Eracle è nell'Ade, il luogo da cui nessun uomo può tornare vivo, e gli amici si sono dimostrati inaffidabili o non sono in grado di dare aiuto (vv. 55-56). Senza essere annunciato, compare sulla scena il coro, composto da vecchi Tebani, diretti verso il palazzo del vecchio Anfitrione, del quale sono stati compagni d'armi nella lontana gioventù. ${ }^{5}$ Il coro intona un breve e lugubre canto funebre, con cui fa riferimento alla presunta e prematura morte di Eracle, rivolgendosi ai figli rimasti orfani, ad Anfitrione ed alla moglie Megara che piange il suo sposo nell'Ade. Al contempo commisera la propria condizione di vecchi

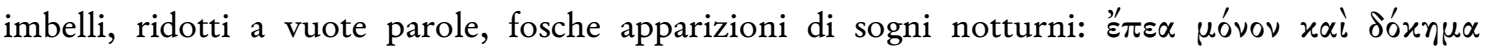

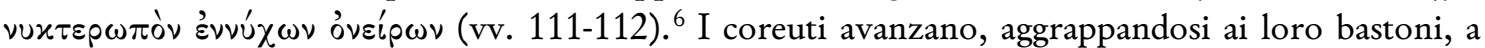
passi tremanti ma non privi di spirito animoso. ${ }^{7}$ Poiché ormai sono incapaci di azione, la loro 'energia' sta nelle parole: sono abili cantori e validi oratori. È significativa la definizione che il coro dà di sé, parlando in prima persona singolare e presentandosi quale "cantore di lamenti, di pianti come il bianco uccello" (vv. 109-110) ${ }^{8}$. Nel paragonare se stesso al cigno, come ci assicura il parallelo

Anfitrione uccise Elettrione in un accesso d'ira durante un alterco per il bestiame: $c f$. anche schol. ad Hom. Il. 14, 323 Erbse.

${ }^{3}$ Il tema della via di salvezza pervade la prima parte della tragedia: si noti l'abbondanza di forme di $\sigma \omega \hat{\omega}^{\prime} \omega$, $\sigma \omega \tau \eta^{\prime} \rho, \sigma \omega \tau \eta \rho^{\prime} \alpha$ (vv. 72, 80, 203, 304, 318, 346, etc.).

${ }^{4}$ Anfitrione collega questo Lico più giovane ad uno più antico legato alla saga tebana (vv. 26-34), il quale non risulta menzionato in altra fonte.

${ }^{5}$ M. Hose, 1991, p. 91: «der Chor verkörpert die Freunde, seine Schwäche und Hinfälligkeit dokumentiert die Ohnmacht». Per il significato dell'autopresentazione del coro dell'Eracle all'interno dell'azione drammatica, vid. M. P. Pattoni, 1990, pp. 43-44 n. 19.

${ }_{6}$ Per esprimere la propria debolezza, i vecchi descrivono se stessi come ombre di qualcuno già morto che si manifestano in sogno: la figura di 'sogno' esprime congiuntamente la condizione di vecchiaia e quella di debolezza, talora associata alla figura di 'ombra'. Questa è un'immagine che radicalizza per gli anziani quella universale di Pi.

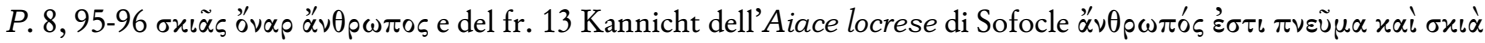
uóvov. È molto vicina a quella usata dai vecchi dell'Agamennone di Eschilo, che considerano la propria esistenza

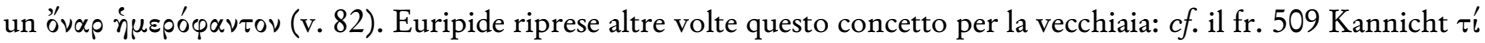

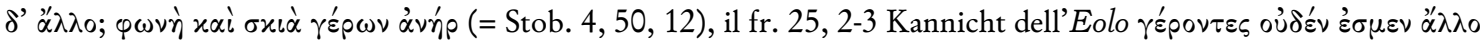

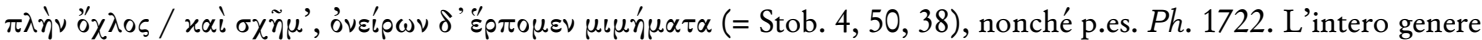

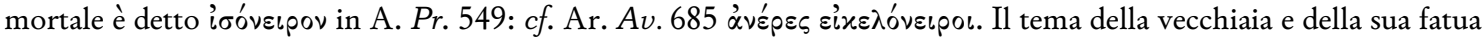
debolezza punteggia tutto il dramma: $c f$. i vv. 268 ss., 312 ss., 436 ss. e soprattutto i vv. 637-700 del secondo stasimo che rievocano il contrasto tra vecchiaia e giovinezza. Per la fragilità senile assimilata a vuote parole, cf. il v. 229

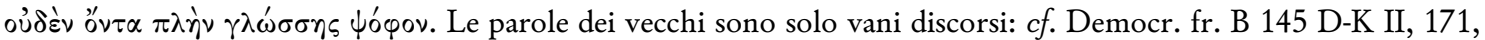

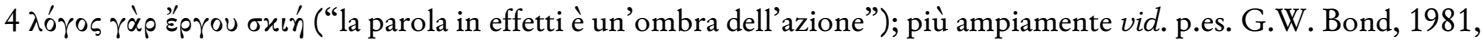
p. 96. U. von Wilamowitz, $1895^{2}$, p. 32 riteneva che questa descrizione dell'età avanzata, pur in uno stile particolarmente nobile e 'barocco', prendesse in prestito «volkstümlichen spruches», elementi proverbiali e di uso colloquiale. Sulla forza stilistica di questo passaggio, vid. anche J. Assaël, 1996, p. 81 e n. 44.

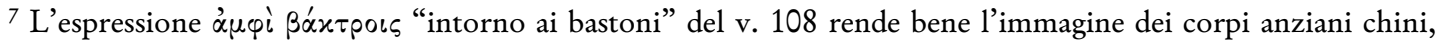
che si aggrappano sui bastoni per camminare: $c f$. G. W. Bond, 1981, p. 94. Anche nelle Vespe di Aristofane gli anziani, che formano il coro, hanno difficoltà a camminare (vv. 230-232), per cui devono sostenersi l'un l'altro come i vecchi dell'Eracle, mentre ricordano le proprie lontane gesta militari (vv. 236-239), un motivo presente anche nei vv. 127-129 dell'Eracle. Affinità sono riconoscibili anche con il Cresfonte (= fr. 448a, 73a-76 Kannicht) in cui il coro di anziani lamenta l'amara condizione della vecchiaia nonché, com'è noto, con i vv. 72-82 dell'Agamennone di Eschilo, possibile fonte d'ispirazione per Euripide, in cui il coro introduce il tema della propria infermità. Per il bastone e le vesti sceniche dei vecchi, $c f$. Poll. 4, 119.

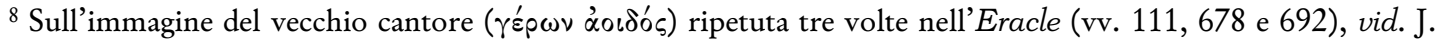
Pòrtulas, 2016, pp. 13-31, in vari contesti letterari. 
Paolo Santé

La parodo dell'Eracle di Euripide: tradizione critica della colometria e del testo

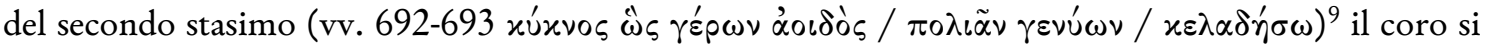
inserisce in una ricca tradizione secondo cui questo uccello, sacro ad Apollo e alle Muse, intonava brevemente il proprio canto prima di morire. ${ }^{10}$ Dunque il riferimento al cigno ha in sé il richiamo a una profezia di morte, ${ }^{11}$ ma rievoca anche la canizie dei vecchi con il suo bianco piumaggio. ${ }^{12}$

Il riferimento incrociato al cigno e al poeta o cantore anziano avrà un suo sviluppo: in $A P 7,30$, 1 Anacreonte morto è detto da Antipatro di Sidone "il cigno di Teo", mentre in AP 5, 134, 3 (= 123, 3 Austin-Bastianini) il filosofo stoico Zenone è definito da Posidippo di Pella "il cigno sapiente". ${ }^{13}$ L'immagine del cigno diventerà topica per identificare un poeta: p.es. Leonida di Taranto la usa per Alcmane in $A P 7,19,1-2$; un epigrammista anonimo per Alceo in $A P 9,571$,

${ }^{9}$ Qui, però, si tratta di un contesto gioia e di lode per la cui analisi, vid. p.es. E. Calderón Dorda 2017, pp. 923. Infatti il cigno è anche in relazione con Apollo, da cui ha ricevuto il dono profetico di intonare il canto più alto e più bello, in prossimità della morte: $c f$. Pl. Phd. $85 \mathrm{a}$. Sulle differenti contestualizzazioni del cigno nei drammi di Euripide, vid. G. Bignardi, 2013, pp. 77-89.

10 In Bacchyl. 16, 6-12 il cigno animale 'canoro' è collegato ad Apollo, dio della musica, per cui vid. anche Alcm. fr. 1, 100-101 Page-Davies. In Alc. fr. 307 Lobel-Page i cigni trainano il carro volante di Apollo, come in Pherecyd. fr. 58 Jacoby, mentre Him. Or. 46 Colonna, 44 ss. ci parla di un carme di Saffo, in cui la poetessa avrebbe cantato il volo di Apollo che, con la cetra in mano e trainato da cigni, saliva sull'Elicona per danzare con le Cariti e le Muse; $c f$. anche Nonn. D. 38, 206. Viceversa in Hom. Il. 2, 459 ss. i cigni sembrano descritti solo come animali "rumorosi" (v. $463 x \lambda \alpha \gamma \gamma \eta \delta o ́ v)$ né sembra contenere riferimenti ad un canto melodioso Ps. Hes. Sc. 316. Per altra via h. Hom. 21, 1 e Pratin. fr. 1, 5 Page-Davies testimoniano una tradizione, secondo cui sarebbe il battito delle loro ali a produrre un suono musicale, mentre Philostr. $\mathrm{Im}$. 1, 9 riferisce un'ulteriore tradizione, per cui il suono si originerebbe dal soffio di zefiro tra le loro piume. È noto che, in età ellenistica, l'immagine del cigno diventerà un topos letterario. Ma si tratta di un tema che si ramifica anche in àmbito mistico: p.es. Pl. R. 620a, nel mito di Er, racconta che l'anima di Orfeo si tramuta proprio in un cigno.

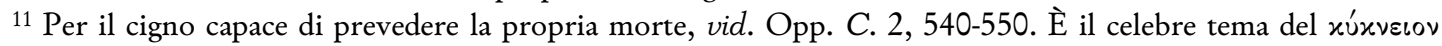
$\tilde{\alpha} \sigma \mu \alpha$, che avrà enorme sviluppo, trattato p.es. anche in una favola di Esopo (174 Chambry $=247$ Hausrath). A. Ag. 1444-1445 è la più antica occorrenza sulla credenza del canto del cigno che ne precede la morte; successivamente vid. E. El. 151, IT 1104, Phä̈th. fr. 78 Diggle (= fr. 773, 34 Kannicht), Ar. Av. 769-772, Ar. Ra. 207, Pl. Phd. 84e-85a, Call. Del. 249. 11 canto del cigno era un problema aperto per l'antica etologia: stando a Plin. HN 10.63 olorum morte narratur flebilis cantus, falso, ut arbitror, aliquot experimentis, nonché al suo probabile

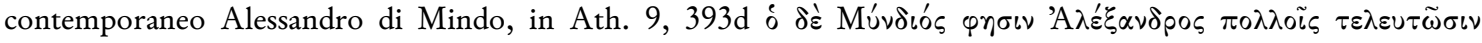

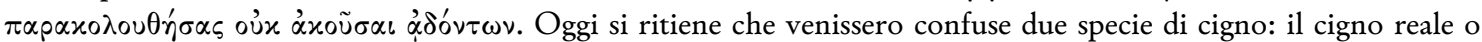
muto (cygnus olor) e il cigno selvatico o canoro (cygnus cygnus). La seconda specie, allorché i polmoni collassano nell'agonia della morte, sembra emettere un lamento sottile e prolungato, per la particolare conformazione della trachea. Tra la fitta bibliografia sull'argomento, vid. p.es. D.W. Thompson, 1895, pp. 104-108; J. Pollard, 1977, pp. 144 ss.; G. Arnott, 1977, pp. 149-153; G. Arnott, 2007, pp.122-124 s.v. kyknos; L. Calero, 2017, pp. 203218.

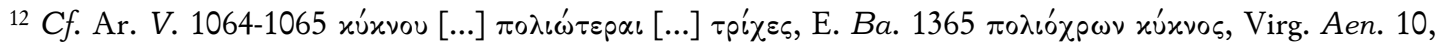
192 canentem molli pluma duxisse senectam, Ov. Tr. 4, 8, 1-2 iam mea cycneas imitantur tempora plumas / inficit et nigras alba senecta comas, Mart. 3, 43, 2 (di un uomo dai capelli tinti) tam subito corvus, qui modo cycnus eras. Il riferimento incrociato alle Graie "dall'aspetto di cigno" in A. Pr. 795 (xuxvópopழoı) con Hes. Th.

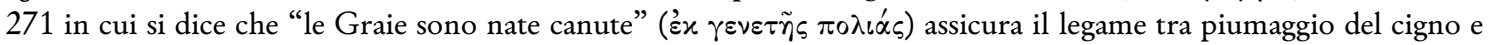
canizie della vecchiaia, che p. es. anche in Sapph. fr. 58, 12 Voigt è detta $\pi \circ \lambda_{\iota}$ ós. Quando i capelli perdono colore, passano da uno stadio intermedio incerto, prima di acquisire il pieno biancore: G. Reiter, 1962, p. 54 ss. precisa

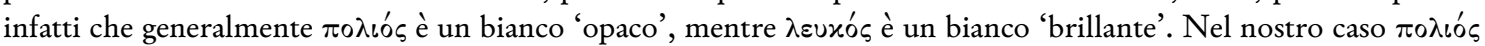
sembra un cromatismo bianco 'opaco', sebbene l'aggettivo contenga in sé anche il grigio 'fumo': $c f$. P. Chantraine,

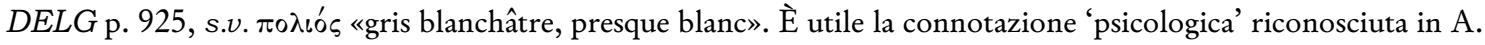
Grand-Clément, 2007, p. 35: «La couleur grisonnante associée à polios trace ainsi la voie d'un passage, marque le franchissement d'une frontière, la marginalisation progressive. Le blanchiment qu'il dénote signifie la perte de vigueur, le glissement vers un état décoloré, atone, une blancheur funeste qui annonce la mort». Per il cigno bianco d'età adulta come termine cromatico di riferimento, $c f$. p.es. E. Hel. 215, in cui Zeus, trasformatosi in cigno adulto,

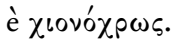

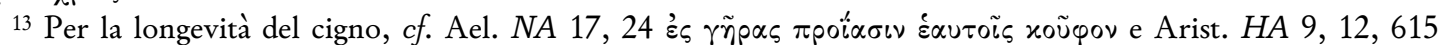

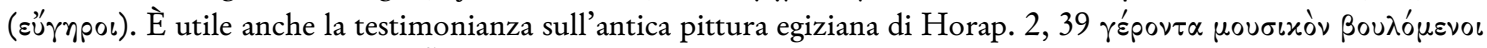

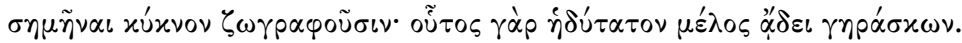


6; Cristodoro per Pindaro in AP2, 382. Una piena assimilazione di se stesso ad un vecchio cigno

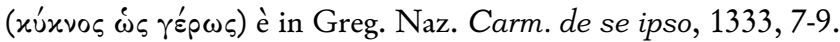

La definizione che il coro dà di sé contiene inoltre il riferimento al yóos e soprattutto allo $i \alpha \lambda \eta \mu \circ \varsigma$ ( $i \eta \dot{\lambda} \lambda \eta \mu \varsigma)$ un termine frequentemente attestato in Euripide, che indica una forma di lamento funebre. Qui potrebbe forse conservare una qualche eco, nella coscienza linguistica di Euripide e del suo uditorio, del pianto di lutto per una morte prematura, con riferimento sia alla presunta morte di Eracle, ${ }^{14}$ sia forse a quella prossima ventura dei suoi figli, su cui si concentra l'epodo.

Per la possibilità di un riferimento ad una morte prematura tra le altre occorrenze euripidee, vid. E. Tr. 1304 in cui Ecuba invoca i figli morti e la sua invocazione è definita dal coro uno ialemos. Inoltre lo scolio a E. Ph. 1033 (1, 358 Schwartz) connette gli ialemoi di madri e ragazze

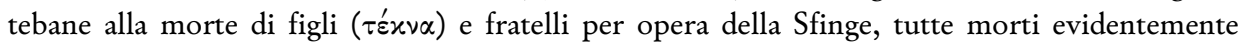
premature. Ma si può anche chiamare in causa E. Rh. 895-897 in cui la Musa definisce il lamento funebre per il proprio figlio uno ialemos. ${ }^{15}$ Secondo una nota tradizione Ialemo, figlio di Apollo e della musa Calliope, morì giovane per una crudele malattia, diventando eponimo di questo canto di lamento. ${ }^{16}$ Il termine perderà la sua specificità per diventare un apparente sinonimo di $\theta \rho \tilde{\eta}$ vos, ${ }^{17}$ forse per un indebolimento del carattere cultuale con cui era connesso in origine. ${ }^{18}$

Successivamente il coro, nel percorrere il cammino verso il palazzo, si paragona ad un puledro aggiogato lungo un pendio. ${ }^{19} \mathrm{Il}$ riferimento al puledro, cioè ad un animale giovane, accresce il contrasto tra la lontana gioventù e l'età avanzata: ${ }^{20} \mathrm{i}$ vecchi non hanno più le forze del giovane puledro, ma ne conservano lo spirito, l'impeto del momento con cui sopraggiungono, rischiando di affaticarsi anzitempo per la loro precaria condizione fisica. ${ }^{21} \mathrm{I}$ coreuti si esortano così ad un aiuto

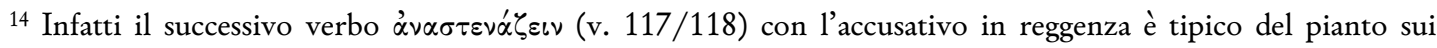
defunti: $c f$. A. Ch. 335 ed E. IT 656.

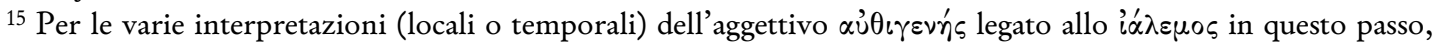
vid. p.es. M. Fantuzzi, 2007, pp. 194-195.

${ }^{16}$ Cf. Ps. Apollod. apud schol. ad Theoc. 10, 41d, p. 235 Wendel; Pi. fr. 128c Snell-Maehler (= fr. 56 Cannatà); schol. ad E. Rh. 895 (2, 343, 13-345,11 Schwartz = 114-115 a Merro); schol. ad E. Or. 1390 (1, 222 Schwartz); schol. ad Pi. P. 4, 313a, p. 140, 8-10 Drachmann. Lino, Imeneo e Ialemo, i tre figli di Apollo e Calliope, identificherebbero tre diversi tipi di $\theta$ p̃̃vos secondo M. Cannatà, 1990, pp. 139-144, ma per lo ialemos come categoria a sé all'interno del genere del $\theta p \tilde{v} v o s, v i d$. già $O$. Jessen, ialemos, $R E 9,11914$, col. 624 ss. Sullo ialemos, vid. anche p.es. EM 463, 15 e Phot. 110, 2. Una notizia trasmessaci dallo scolio a E. Ph. 1033 in PWürzb. 1 sembra collegare la morte di Ialemo al suo matrimonio: per le varie possibili spiegazioni, vid. H. Essler \& D. Mastronarde \& K. McNamee, 2013, p. 78.

17 Probabilmente in età classica, certamente in età ellenistica, con il termine $\theta \rho \tilde{p} v o \varsigma$ si intendeva il canto funebre composto preventivamente da un poeta e poi eseguito da un coro, al momento delle esequie o durante cerimonie commemorative. Si ritiene invece che, nella cultura epica, il róos fosse un pianto di donne a carattere spontaneo, regolato da precise norme rituali e legato al cordoglio di congiunti, mentre il $\theta$ p ñvos fosse un canto più elaborato intonato dagli aedi, secondo l'esempio dei funerali di Ettore in Hom. Il. 24, 719-776 e di Achille in Hom. Od. 24, 57 ss. Per l'uso e il significato dei termini yóos e $\theta$ p ñvos in tragedia, con opinioni non sempre simili tra loro, vid. p.es. M. Alexiou, 2002, p. 102 ss., N. Loraux, 2002, pp. 58-59, L. A. Swift, 2010, pp. 303-304, N. Weiss, 2017, pp. 243-266.

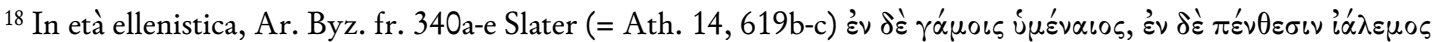
considera lo ialemos un sinonimo del $\theta$ p $\tilde{\nu} \circ \varsigma$, testimoniandoci l'avvenuta generalizzazione del termine: più ampiamente vid. R. Palmisciano, 2017, pp. 93-95. Altre occorrenze del termine iń $\lambda \varepsilon \mu \circ \varsigma$ ( $\left.{ }^{\prime} \alpha \lambda \varepsilon \mu \circ \varsigma\right)$ in tragedia sono A. Supp. 114-116, Ch. 424 con l'hapax in $\lambda \varepsilon \mu \iota \tau \tau \rho^{\prime} \alpha \varsigma$ (= Hsch. ı 367), E. Supp. 281, Ph. 1033-1034, Tr. 604 e 1304, Or. 1390, Rh. 895, fr. $953 \mathrm{~m}$ Kannicht.

${ }^{19}$ In questo punto il testo è tràdito in maniera corrotta, ma il senso complessivo sembra deducibile: vid. oltre.

${ }^{20}$ Lo nota p.es. L.A. Swift, 2010, p. 123, n. 39.

21 Per la sollecitudine del coro nell'arrivare, cf. p.es. A. Pr. 129 ss. E. Hec. 98 ss. Questo spirito sollecito contrasta con la camminata lenta e cadenzata dall'appoggio sul bastone propria di un anziano, accentuata e quasi scandita dall'allitterazione in occlusiva labiale $(\pi)$ : vv. 119 e ss. [...] $\pi \rho \circ x_{\alpha}^{\prime} \mu \eta \tau \varepsilon \pi \delta^{\prime} \delta \alpha[\ldots] \pi \rho o ́ \varsigma ~ \pi \varepsilon \tau \rho \alpha \tilde{o} \circ \lambda \varepsilon^{\prime} \pi \alpha \varsigma[\ldots]$ $\pi \tilde{\omega} \lambda$ ov. 
Paolo Santé

La parodo dell'Eracle di Euripide: tradizione critica della colometria e del testo

reciproco, affinché il più forte guidi i passi del più debole, nel ricordo ormai lontano delle comuni e gloriose gesta del passato. ${ }^{22}$

\begin{abstract}
Alcune questioni sceniche restano dibattute. Innanzitutto se il coro, nel fare il suo ingresso, salga realmente lungo una qualche pendenza per accedere alla scena oppure se il riferimento alla 'salita' sia puramente convenzionale e immaginario. ${ }^{23} \mathrm{Un}$ altro aspetto discusso è se il coro sia diviso o meno in gruppi che entrano separatamente, per poi riunirsi. Nel secondo caso, il coro entrerebbe in due tempi. La strofe verrebbe cantata da una prima metà dei coreuti mentre, con l'inizio dell'antistrofe, il resto dei coreuti si rivolgerebbe agli altri esortandoli a mantenere un passo adeguato alla loro età finché, con l'inizio dell'epodo, il coro riunito rivolgerebbe la propria

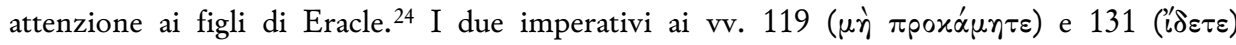
potrebbero collimare con due gruppi distinti di coreuti, ma l'ipotesi a cui si dà più credito è che il coro si rivolga a se stesso sollecitandosi, secondo un uso che sembra testimoniato in questa e in altre tragedie di Euripide. ${ }^{25}$
\end{abstract}

Infine il coro si volge a guardare i figli di Eracle, come se li indicasse: ${ }^{26}$ i loro occhi ardenti (v. 131 a үop $\tilde{\omega} \pi \varepsilon \varsigma)$ ricordano quelli del padre, ${ }^{27}$ in un'inquietante anticipazione dello sguardo furioso di Eracle impazzito ( $c f$. i vv. 868 e 990), nonché gli occhi delle serpi contro Eracle in fasce (v. 1266). La sventura grava su di loro, ma la loro grazia infantile è intatta. ${ }^{28}$ La morte di questi giovani sarà una grave perdita per la Grecia tutta, a cui il coro si rivolge con un'accorata allocuzione (vv. 135137).

In altra sede, ho provato ad argomentare come il testo tràdito dei versi iniziali della parodo (vv. 107 119) ampiamente discusso e corretto si presenti del tutto ammissibile. ${ }^{29}$ Numerosi altri luoghi sono stati discussi e variamente emendati da una lunga e autorevole schiera di filologi. Li ridiscuto in questa sede avanzando, dove riterrò di avere trovato argomenti adeguati, una difesa del testo e della colometria tràditi dai codici Laur.gr. 32, 2 (L) e Vat. Pal.gr. 287 + Conv. Soppr. 172 (P).

I due codici sono oggetto di una lunga controversia, relativa ai rapporti che intercorrano tra loro. Per le tragedie 'alfabetiche', essendo certo che $\mathrm{L}$ non sia copia di $\mathrm{P}$, resta fonte di discussione se $\mathrm{P}$ sia 'fratello' di L, cioè copiato dal medesimo antigrafo, oppure se $\mathrm{P}$ sia stato copiato direttamente da $\mathrm{L}$, ed in questo caso se per tutto il testo euripideo o soltanto in alcune sezioni. Nell'uno e nell'altro caso, si potrebbe supporre un rapporto più complesso per l'eventuale presenza di una copia intermedia. Gli studiosi si sono schierati per l'una o per l'altra interpretazione, sulla base di due lavori fondamentali, quello di A. Turyn, 1957 che pensava si trattasse di copie autonome di un esemplare comune, e quello di G. Zuntz, 1965 che, per i drammi 'alfabetici', riteneva $\mathrm{P}$ copia di L dopo una serie di correzioni apportate da Demetrio Triclinio $\left(\operatorname{Tr}^{1}\right)$, prima di ulteriori correzioni $\left(\operatorname{Tr}^{2}\right.$ e $\left.\operatorname{Tr}^{3}\right)$ riconoscibili dal diverso colore dell'inchiostro. Quanto all'Eracle, G. Zuntz, 1965, pp. 87-88 precisa quanto segue:«It is true that, here again, black where shading towards brown may be pratically identic with a dark

22 Il riferimento è alla battaglia contro i Tafi (vv. 60-61 e v. 1080): Anfitrione alla testa dei Tebani aveva sconfitto gli abitanti di Tafo in Acarnania.

${ }^{23}$ Vid. G.W. Bond, 1981, p. 92 che sintetizza gli aspetti salenti delle due questioni.

${ }^{24}$ Cosi L. Parmentier, 1923, p. 26 ma M. Kaimio, 1970, p. 132: «There is no proof for this arrangement».

$25 \mathrm{Vid}$. M. Kaimio, 1970, pp. 131-137 per vari esempi euripidei: v. 126 e poi HF 875, Supp. 271-274, Tr. 1235,

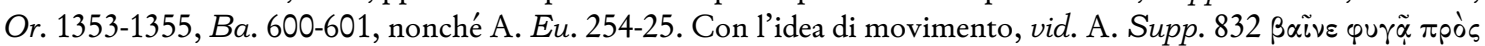
$\dot{\alpha} \lambda x \alpha \alpha$. Un'interpretazione alternativa si legge in J. S. Lasso de la Vega, 1990, p. 20.

${ }^{26}$ Notevole, in questo senso, la deissi $\alpha^{\prime \prime} \delta \varepsilon[\ldots] \alpha \cup \gamma \gamma \alpha i$ (vv. 131a-132).

${ }^{27} \mathrm{Ma}$ anche quelli di Eracle bambino: $c f$. Pi. fr. 52u, 13 Maeher (= fr. S1 Rutherford). Si noti la posizione enfatica di $\pi \alpha \tau$ pò $\zeta$ nella frase.

28 Più logicamente il termine $\chi \alpha ́$ ṕs (v. 134) fa riferimento alla bellezza infantile e non alla gratitudine degli anziani, che non hanno ragioni di riconoscenza diretta nei confronti di questi ragazzini, sebbene in loro riviva Eracle, come opportunamente osserva U. von Wilamowitz, $1895^{2}$, p. 36

${ }_{29}$ P. Santé, 2019, pp. 133-138. In L un piccolo spazio dopo $\mu \varepsilon ́ \lambda \alpha \theta \rho \alpha$ è dovuto all'usus scribendi e non compare nell'antistrofe. L'incongrua divisione con un docmio iniziale è reperibile a ritroso sin da B. Heath 1762, p. 143 «antispasticus hemiolius». 
variety of brown used by $\mathrm{Tr}^{3}$, in places which lack the now familiar red glow; even so, the distinction is hardly ever in doubt when attention is paid to the style of writing and neighbouring passages wich will often show to wich working process any particolar Triclinan contributon belongs». Infatti anche K. H. Lee, 1988, p. V scrive, con riferimento all'Eracle: «tres series Tricliniarum secernere est opus dignum Lynceo». Tra i recentiores sono di un certo interesse il Laur. plut. 31,1, apografo di L di metà XV secolo, su cui vid. più ampiamente A. Turyn, 1957, p. 364, e il Par. gr. 2817 degli inizi del XVI secolo, anch'esso apografo di L. Al v. 107 nel Laur.

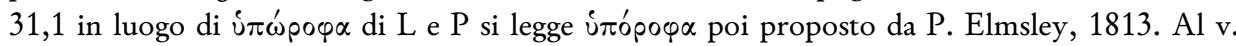
123 in luogo di pépov di L e P, nel Laur. 31,1 e nel Par. gr. 2817 post correptionem si legge $\varphi$ ह́ $\rho \omega \nu$, poi proposto da vari studiosi nelle loro ricostruzioni del testo: p.es. J.A. Hartung, 1849, A. Nauck, $1871^{3}$, U. von Wilamowitz, 1895². Il Par. gr. 2714 del XVI secolo, per una parte copia diretta di L per l'altra copia del Laur. 31,1, al v. 114/115 presenta (incidentalmente?) una

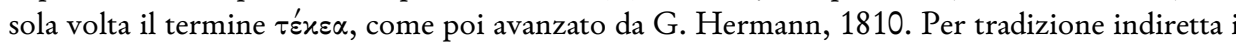
vv. 111-112 sono tramandati in Plu. Comm. not. 1066c: il testo di Plutarco differisce in due

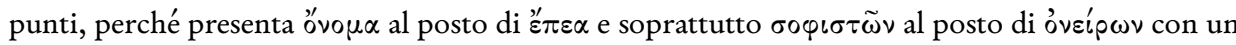
evidente intento parodico. Infine i vv. 107-109 sembrano trovare un'eco in Luc. Pod. 58-59

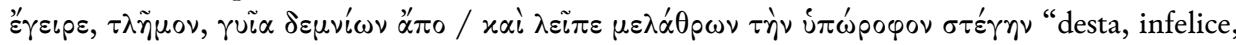
le membra dal giaciglio / e lascia la stanza e il tetto della casa”. Il luogo è stato ritenuto utile da chi ha sostenuto che il coro di anziani, nel fare il suo ingresso, debba indicare il luogo dal quale arriva e non quello verso cui arriva. Di fatto, però, la sezione che va dal v. 54 al v. 82, riprendendo il monologo paratragico del podagroso e l'apostrofe al bastone, mescola liberamente espressioni e vocaboli di ascendenza epica e tragica. ${ }^{30}$

Riporto di seguito il testo greco, con traduzione e analisi metrica conforme all'assetto colometrico e alla paradosis testuale trasmessi dai manoscritti. Eventuali variazioni sono segnalate nell'apparato testuale e in quello colometrico, che segue l'interpretazione metrica. ${ }^{31}$

\section{Testo e colometria}

$$
\begin{aligned}
& \sigma \tau \rho \text {. نं } \pi \omega^{\prime} \rho \circ \varphi \alpha \mu \varepsilon^{\prime} \lambda \alpha \theta^{\prime} \rho \alpha x \alpha i{ }^{\prime} \gamma \varepsilon \rho \alpha i \dot{\alpha} \\
& \delta \text { 'suvi' } \alpha \mu \varphi i \beta^{\prime} \alpha x \tau \rho \circ \iota s
\end{aligned}
$$

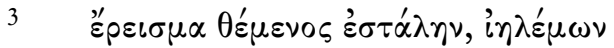

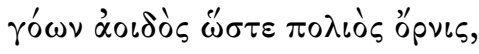

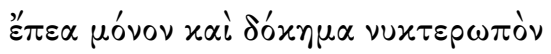

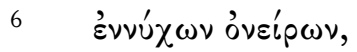

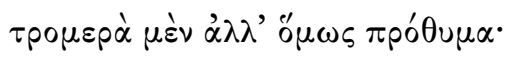

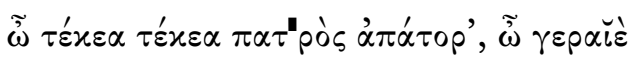

$$
\begin{aligned}
& 9 \sigma u^{\prime} \tau \varepsilon \tau \dot{\alpha} \lambda \alpha \iota \nu \alpha \mu \tilde{\alpha} \tau \varepsilon \rho, \ddot{\alpha}
\end{aligned}
$$

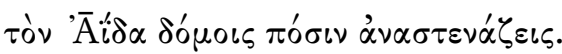

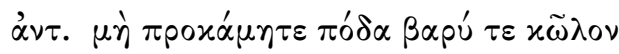

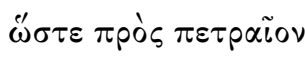

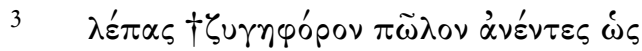

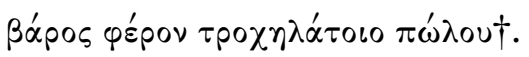

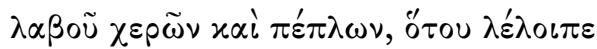

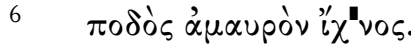

30 Più ampiamente vid. P. Santé, 2019, p. 136, n. 6.

${ }^{31}$ Per le cui edizioni e i commenti, vid. la Bibliografia finale. 
Paolo Santé

La parodo dell'Eracle di Euripide: tradizione critica della colometria e del testo

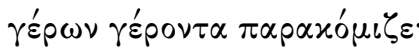

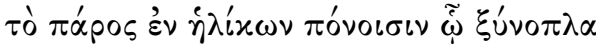

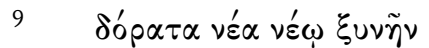

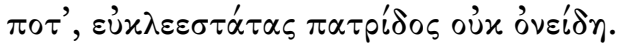

$129 / 130$

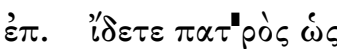

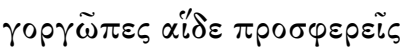

$131 \mathrm{a}$

$3 \quad$ o $\mu \mu \alpha \alpha^{\prime} \omega \nu \alpha \dot{\gamma} \gamma \alpha \dot{l}$,

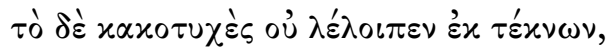

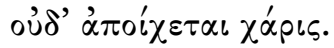

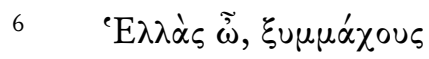

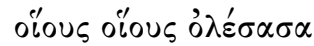

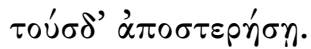

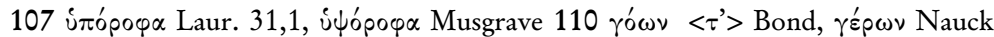

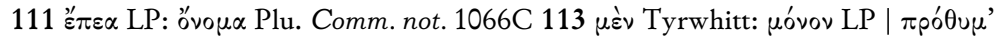

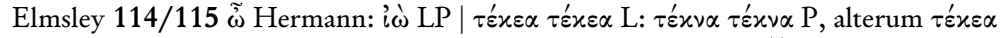

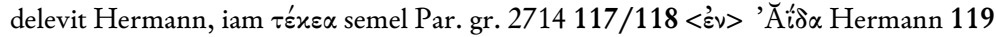

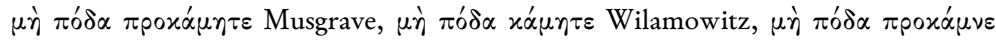

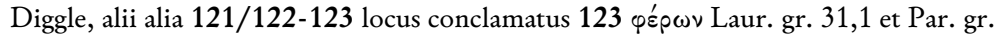

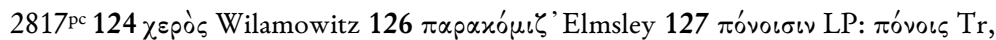

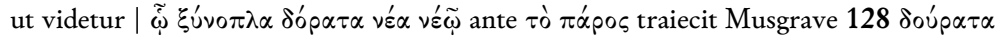

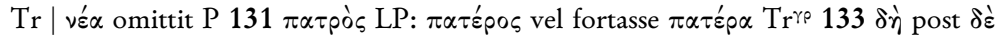

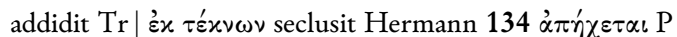

\section{Analisi metrica e apparato colometrico}

str./ant.

\begin{tabular}{|c|c|c|}
\hline $107 \sim 119$ & $a u k y \quad t \quad k t k \mid k$ & ia ( cho) ithyph \\
\hline $108 \sim 120$ & l $k|k| k \mid$ & ithyph \\
\hline 109 121/122 & ${ }^{3} k$ | tk y k | a u k l k l & $3 \mathrm{ia}^{32}$ \\
\hline $110 \sim 123$ & $k|k| k \mid k y$ & $3 \mathrm{ia}^{\wedge}$ \\
\hline $111 \sim 124$ & 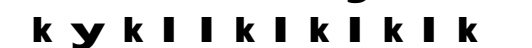 & ia $2 \mathrm{tr}$ \\
\hline $112 \sim 125$ & ${ }^{6} \mathrm{t} \quad \mathrm{k}|\mathrm{k}| \mathrm{l}$ & ithyph \\
\hline $113 \sim 126$ & $\begin{array}{llllll}k & y & k & k & k & k U H\end{array}$ & 2ia hypercat \\
\hline $114 / 115 \sim 127$ & $u k k$ k $y \quad k \quad k y$ & $3 \mathrm{ia}$ \\
\hline $116 \sim 128$ & ${ }^{9} k \dot{k} k t k|k|$ & $2 \operatorname{tr} \wedge$ \\
\hline $17 / 118 \sim 129 / 130$ & $k|k| k|k k \| k| k|| H$ & $2 \mathrm{ia} \operatorname{tr}$ \\
\hline
\end{tabular}

ep.

\begin{tabular}{|c|c|c|}
\hline 131 & $k k k|k|$ & hypodo vel penthem ${ }^{\text {tr }}$ \\
\hline $131 \mathrm{a}$ & | | k l k | k | & $2 \mathrm{ia}$ \\
\hline 132 & $3|k|||$ & hypodo vel penthem ${ }^{\text {tr }}$ \\
\hline 133 & $k \dot{k} k k \dot{k} k|k| k|k| k \mid$ & $3 \operatorname{tr} \wedge$ \\
\hline 34 & l k | k | k u bb & $2 \operatorname{tr} \wedge$ \\
\hline
\end{tabular}

${ }^{32} \mathrm{Il}$ testo manoscritto dell'antistrofe è corrotto e, come si vede, non è riconducibile allo schema del 3 ia della strofe. 
135

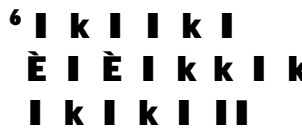

en ${ }^{\mathrm{b}}$

ithyph

MARGINALIA METRICA TRICLINIANA $107 \sigma \tau \rho\left(\circ \varphi \eta^{\prime}\right) \cdot \chi \circ \rho \iota \alpha \mu \beta \iota x \alpha^{\prime}:$

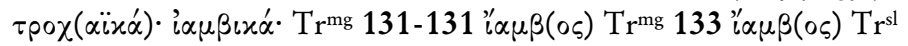

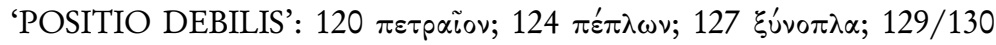

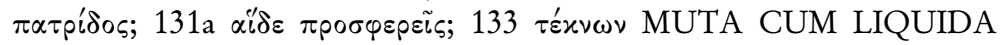

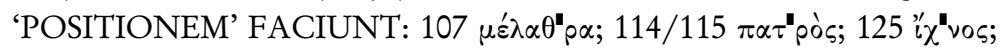

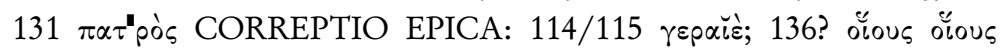
DIAERESIS: $117 / 118$ 'Aî́ $\delta \alpha$ NOTABILIA: $117 / 118$ 'Āî́ $\delta \alpha$

STR./ANT.

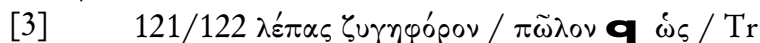

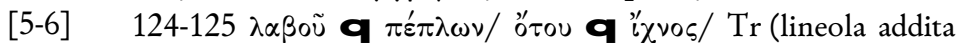
inter $\lambda \varepsilon \dot{\varepsilon} \lambda o เ \pi \varepsilon$ et $\pi \circ \delta \dot{o} \varsigma)$

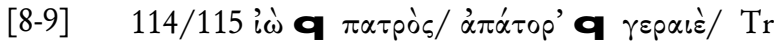

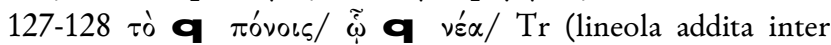
$\xi u ́ v o \pi \lambda \alpha$ et $\delta$ úp $\alpha \tau \alpha)$

[9-10] 117/118 unum colon PTrac (lineola addita inter tó

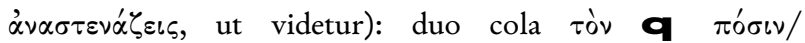

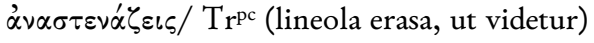

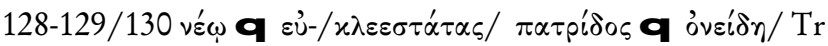

EP.

[1-2] 131-131a sic PTrac: unum colon $\operatorname{Tr}^{\mathrm{pc}}$

\section{Traduzione}

Alle stanze coperte dal tetto, al vecchio letto, sul bastone aggrappandomi a sostegno mi avviai, di lamenti, di pianti cantore come il bianco uccello, solo parole e oscura parvenza

di sogni notturni, tremolante nondimeno animoso. O figli, figli orfani del padre! O vecchio, e tu madre sventurata che piangi lo sposo nelle case dell'Ade!

Non sfinite anzitempo il piede e la gamba pesante, su per petrosa altura come †un puledro aggiogato liberandoli, sapendo che (la gamba) porta il peso di un puledro che muova le ruote di un carro. $\dagger^{34}$

$5 \quad$ Tieni per le mani e per il mantello chi lascia una debole orma del piede. Vecchio accompagna un vecchio! Tempo fa, nelle gesta dei coetanei, con lui giovane le giovanili lance e le altre armi un di furono in comune, di una patria tanto gloriosa non indegne.

33 I cretici dell'epodo (colon 6) vanno intesi come 'esasemi', cioè protratti a trochei: $c f$. B. Gentili \& L. Lomiento, 2003, pp. 17-18 e 220-223. La prassi è supponibile nei canti di ritmo giambo-trocaico dell'ultimo Euripide, come dimostrano gli esempi nella trattazione del Pap. Oxy. $9+268752$.

${ }^{34}$ Per le problematiche connesse al testo, corrotto in questo punto, e alla sua traduzione, vid. oltre. 
Paolo Santé

La parodo dell'Eracle di Euripide: tradizione critica della colometria e del testo

Guardate, così simili a quelli del padre

sguardo di Gorgone sono queste

luci degli occhi.

La sventura non si è allontanata dai figli.

Non svanisce la grazia.

Grecia -ah!- quali, quali

alleati, se perdi loro,

ti verranno tolti!

\section{Tradizione e critica della colometria}

Demetrio Triclinio, nel suo lavoro di studio e revisione dei foll. $97^{\mathrm{r}-\mathrm{v}}$, non ha riconosciuto la responsione o comunque non è riuscito a ricostruirla: manca infatti il marginale $\dot{\alpha} v \tau(\iota) \sigma \tau \rho\left(\circ \varphi \eta^{\prime}\right) .{ }^{35} \mathrm{I}$ suoi numerosi interventi hanno modificato significativamente colometria e testo originali. Una volta individuato il brano lirico, qualificato in margine con la dicitura $\sigma \tau \rho\left(\circ \varphi \eta^{\prime}\right)$ e segnalato il suo inizio

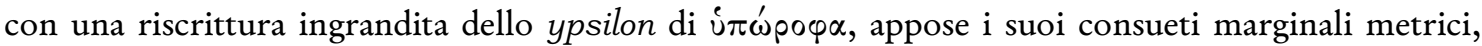

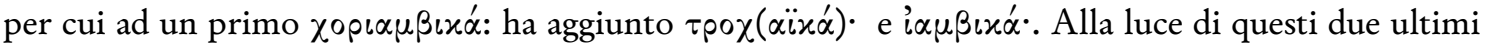
marginali, tutto il suo lavoro appare piuttosto coerente.

Al vv. 114/115 ha diviso in due il colon unico, apponendo un dicolon dopo $\pi \alpha \tau$ pò $s$ in un minimo

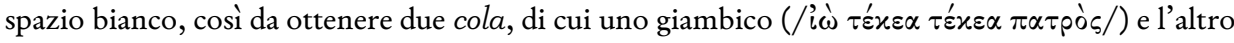

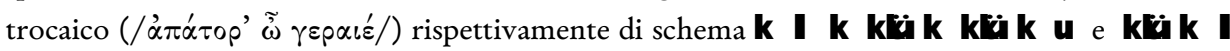

k l k. In P si legge $\tau \varepsilon^{\prime} \varkappa v \alpha \tau \tau_{\varepsilon}^{\prime} \varkappa \alpha$ e il colon è unico. Apparentemente è solo un errore del copista, ${ }^{36}$ perché non sembra cogliersi una ratio metrica alternativa, sia che ì̀ venga considerato nel metro, sia che venga considerato extra metrum. Al v. 116 Triclinio rafforza la fine di colon aggiungendo un dicolon in uno spazio bianco già adeguato. Al v. 117/118 è intervenuto sulla colometria. Il verbo $\alpha \nu \alpha \sigma \tau \varepsilon v \alpha \dot{\alpha} \zeta \varepsilon \iota \varsigma$ è separato dal cambio di colonna, ma nel bianco intercolonnare mi sembra scorresse una linea che lo univa al colon precedente, perché il tratto

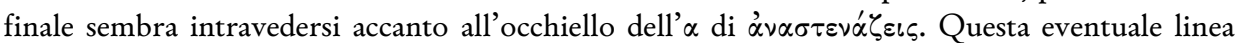
sarà stata opera di Triclinio, che prima l'ha inserita e poi l'ha erasa, ${ }^{37}$ isolando alla fine

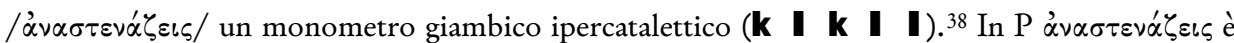

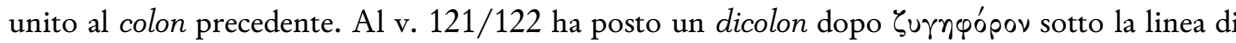

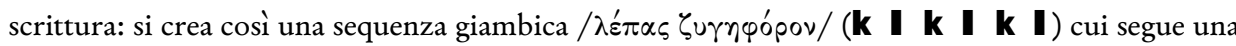

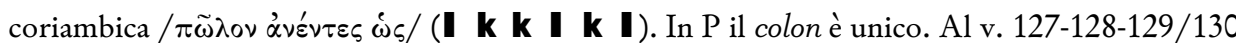

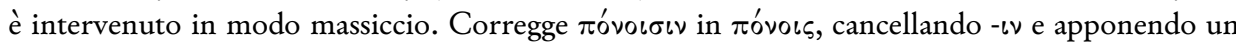

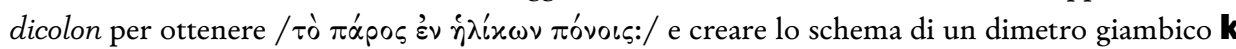
k k̈ k | k | k |. In P c'è lo stadio precedente alle sue modifiche. Unisce con una lineetta $\xi_{u}^{\prime} v 0 \pi \lambda \alpha$ a ciò che segue, corregge $\delta o^{\prime} \rho \alpha \tau \alpha$ in $\delta o u ́ p \alpha \tau \alpha$ e aggiunge un dicolon dopo vé $\alpha$ per ottenere

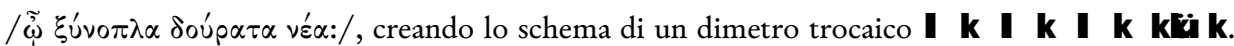
In $\mathrm{P}$ compare lo stadio precedente alle sue modifiche. Poi, dopo avere forse cancellato un dicolon dopo $\xi u v \tilde{\nu} \nu$, allunga verso sinistra il tratto superiore del $\pi$ di $\pi \circ \tau$ ' e appone un dicolon dopo $\varepsilon \dot{v}-$ sotto la linea di scrittura, ottenendo il colon / vé giambico k | k | k |. In P c'è lo stadio precedente a queste modifiche, con il dicolon dopo

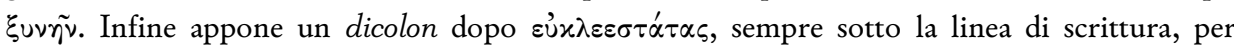

35 A contribuire al mancato riconoscimento della responsione furono, con ogni probabilità, sia il precario stato di conservazione della colometria dai vv. 128 ai vv. 129/130, sia alcuni punti del testo di problematica trasmissione,

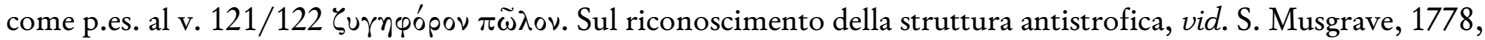
p. 479: «Haec Ode in Editis omnibus tanquam Monostrophica exhibetur, sed re vera Antistrophicam esse monuit me Tyrwhittius».

${ }^{36}$ I copisti degli apografi di L non sono caduti nel medesimo errore di lettura: il Par. gr. 2887 fol. 49r, il Par.

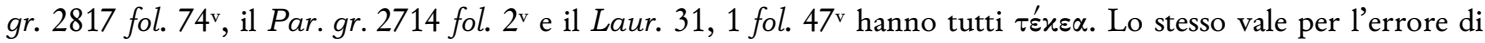
pronuncia itacistica $\alpha \dot{\gamma} \eta \dot{\chi} \chi \tau \alpha \iota$ in P al v. 137 da cui sono esenti gli apografi di L.

37 Pur conservando qualche dubbio, arriverei a questa conclusione.

${ }^{38}$ Questa divisione colometrica è senza dubbio erronea perché, se riportata nell'antistrofe, causerebbe una brevis

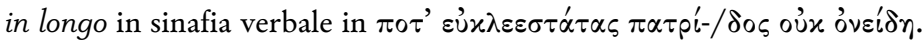




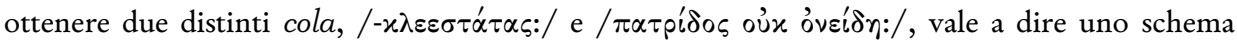
giambico ed uno trocaico: $k|k|$ e k k k

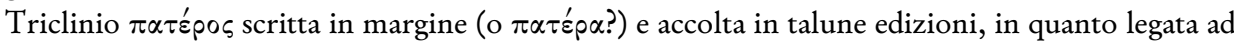
un'interpretazione giambica forzosa, come lui stesso chiarisce, scrivendo ' $\alpha \mu \beta(\circ \varsigma)$ a margine. Triclinio prima ha aggiunto e poi ha cancellato un dicolon di cui si intravedono le tracce sbiadite,

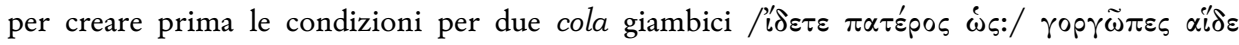

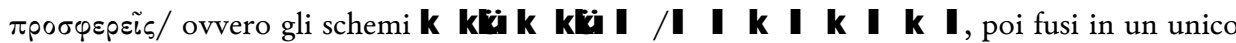

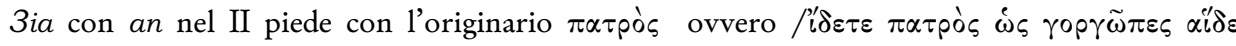

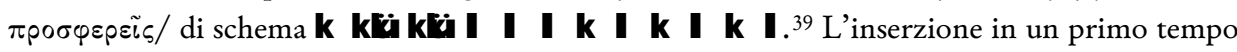
del dicolon dopo $\dot{\omega}_{\varsigma}$ (che compare anche in P) sembra giusta, in quanto corregge la conflazione di due cola. Al v. 133 ha il medesimo scopo l'inserzione di Triclinio di un $\delta \dot{\eta}$ in $/ \tau \dot{o} \delta \dot{\varepsilon} \delta \dot{\eta}$

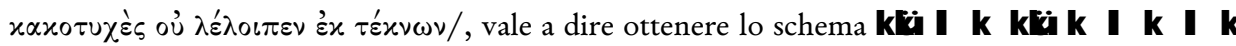
| $k \mid$, perché lui stesso scrive nell'interlinea ' $\alpha \mu \beta(\circ \varsigma)$, in questo caso un 3ia con an nel I piede.

Conformemente alla colometria manoscritta, la versificazione dell'epodo è prevalentemente trocaica ed è diversa della diade antistrofica, che si presenta come una mescolanza giambico-trocaica: $c f$. soprattutto i cc. 1,5 e 10. Nell'epodo si può riconoscere un'accentuazione del ritmo trocaico, ${ }^{40}$ che secondo l'interpretazione di molti, sottolineerebbe l'eccitazione del coro nel vedere i piccoli figli di Eracle. ${ }^{41}$ Nella diade antistrofica, nello stretto intreccio giambico-trocaico la presenza dei giambi è riconducibile al genere dello ialemos ${ }^{42}$ e la commistione con i trochei è tipica dello stile di Euripide, quando vuole calcare su un tono lugubre e funereo, finanche lamentevole, come nella strofe: ulteriori significativi esempi di questa versificazione mista di giambi e trochei, in un contesto trenodico, sono Tr. 1302-1316 1317-1332 e Ph. 1018-1042 1043-1066. ${ }^{43}$

La cornice metrico-ritmica teorica è l'epiploce diadica trisema, cioè l'intreccio di giambi e di trochei, su cui vid. Heph. Ench. 77, 4 e più ampiamente schol. B ad Heph. Ench. 257,1 ss. e

39 La competenza metrica di Triclinio, a lungo esercitata sulle sezioni in trimetri giambici, notoriamente lo indusse a 'fabbricare' altri trimetri ex novo nelle sezioni in metri lirici. La presenza degli anapesti, anche in sede

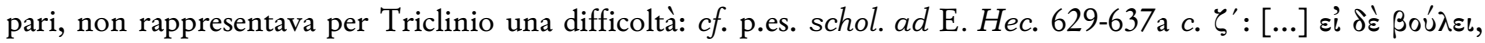

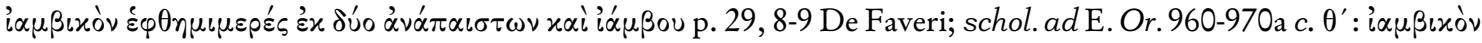

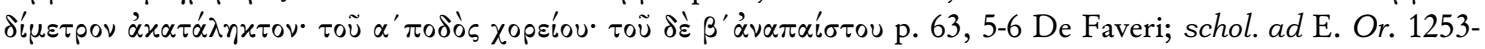

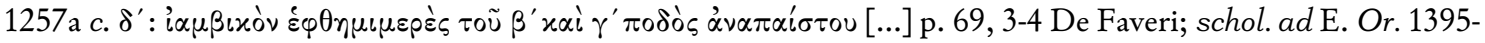

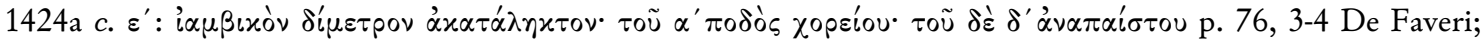

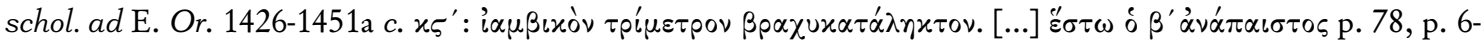
8 De Faveri.

$40 \mathrm{Vid}$. già U. von Wilamowitz, $1895^{2}$, p. 26: «Ein strophenpar in iambischem maße und eine trochäische epode. Der ganz ungewöhnliche umschlag des rhythmus erklärt sich dadurch, daß die strophen auf dem zuge des chores gesungen werden, die epode, nachdem er seinen standort erreicht hat»; ma anche G.W. Bond, 1981, p. 99: «The chorus switch to trochees to mark their excitement at seeing the children and perhaps the end of their climb» nonché S. Barlow, 1996, p. 130 e J. Assaël, 1996, p. 79: «En effet le rythme trochäque s'adapte parfaitement à la situation: il imite les battements syncopés du choeur des vieux choreutes essouflés et affolés».

${ }^{41} \mathrm{Ma}$ si dovrebbe tenere conto del fatto che il coro compiange i piccoli figli di Eracle già prima: $c f$. il v. 114/115.

42 Cf. p.es. A. Supp. 112-116 123-127 con relativo commento di L. Lomiento, 2008a, p. 70 e poi di L.

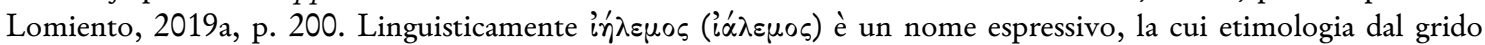
giambico iń (í́) è stata diffusamente sostenuta: vid. p.es. P. Chantraine, DELG, p. 452 e U. von Wilamowitz, $1895^{2}$, p. 30, e cf. A. Suppl. 115-116, E. Phoen. 1033-1037; per un'origine tracio-frigia del termine vid. R. Beekes \& L. van Beck, $E D G$, s.v. ía $\lambda \varepsilon \mu \circ \varsigma$, p. 572, con i relativi riferimenti bibliografici. È interessante che, non di raro, risulti un legame con i popoli barbari: cf. p.es. A. Ch. 424, E. Tr. 604 e 1304, Ph. 1303-1304, Or. 1390.

43 Un'analisi della colometria di questi ultimi versi è in B. Gentili \& L. Lomiento, 2003, pp. 127-128. La coincidenza della sezione più uniformemente giambica con il richiamo agli iēlemoi delle madri e delle ragazze, dovuti alle uccisioni della Sfinge, e con il grido di lamento giambico (inińnıv) appare molto significativa; $c f$. p.es. anche A. Supp. 114-115. 
Paolo Santé

La parodo dell'Eracle di Euripide: tradizione critica della colometria e del testo

Mar. Vict. GL 6, 63 ss. e 94, 6 ss. ${ }^{44}$ Credo possa non essere un caso che Hsch. $x$ 816 Latte, alla

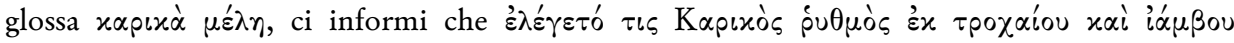

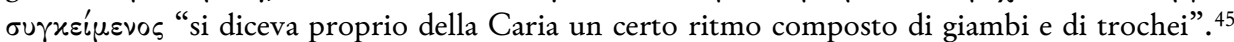

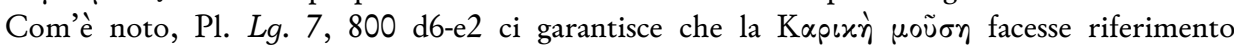
essenzialmente ai lamenti funebri. ${ }^{46}$ Ebbene in Ar. Ra. 1302-1303 Eschilo ci elenca, in esagerata

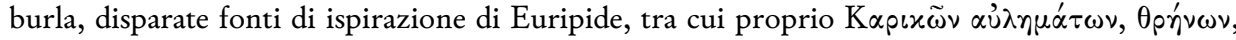
$\chi \circ \rho \varepsilon\left\llcorner\widetilde{\omega} \nu\right.$ "le melodie per flauto, i lamenti funebri, le danze dei Cari". ${ }^{47}$ Per i lamenti funebri, verrebbe alla luce un nesso con la versificazione mista di giambi e trochei di questa parodo, nonché per i già citati $P h .1018-1042$ 1043-1066 e Tr. 1302-1316 1317-1332. Sembra un influsso orientaleggiante.

${ }^{44}$ Proprio la sussistenza dell'epiploce mi induce a escludere che p.es. le sequenze dei vv. 116, 133 e 134 possano essere acefale e avere ritmo giambico invece che trocaico.

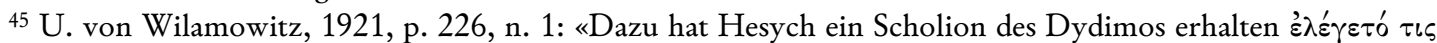

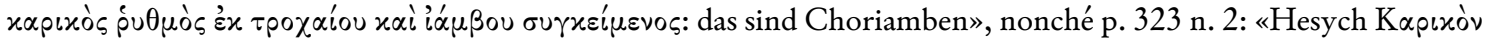

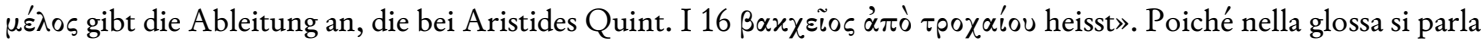

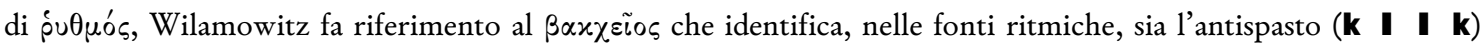
che il coriambo $\left(\begin{array}{l}\mid \\ k\end{array} \quad \mid\right.$ ). Heph. Ench. p. 10, 13 inserisce anche il coriambo tra i 'piedi' composti di quattro sillabe, da sei fino a otto tempi, insieme ai 'piedi' semplici di tre tempi, appunto il trocheo $(\mid k)$ e il giambo $(k \mid)$

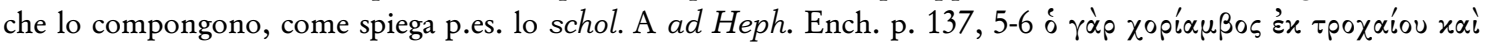

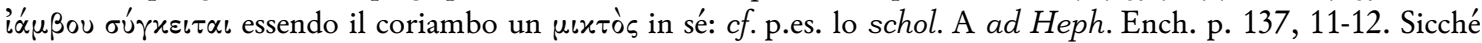
l'interpretazione di Wilamowitz è possibile. Osserverei però che, pur nella terminologia dei metricologi e non dei ritmicologi, sia Heph. Ench. 12, 15, che Choerob. in Heph. Ench. 219, 13, che lo schol. B ad Heph. Ench. 303, 27, che Anon. Ambr. p. 229, 20-22 Studemund (con la variante xápıos) testimoniano che xapıxós era un altro

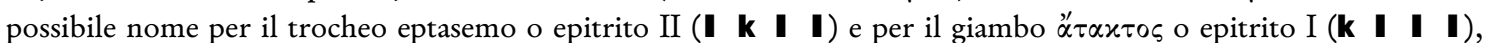
certificando una relazione con i trochei e i giambi, non con i coriambi. Peraltro $\sigma u ́ \gamma x \varepsilon \iota \tau \alpha \iota$ può essere utilizzato anche per definire 'composti' con riferimento ai metra: cf. p.es. schol. ad Pi. P. 1 str. 4, p. 14, 5-7 Tessier

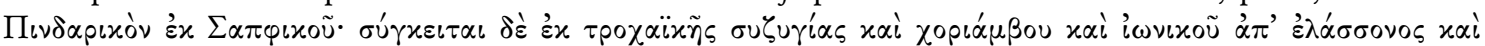
$\sigma \cup \lambda \lambda \alpha \beta \tilde{\eta}$ s, ma sono equivalenti anche gli scholl. ad P. 1 ep. 14, p. 15, 7-8; P. 2 ep. 13, p. 16, 3-5; N. 1. str. 7, p. 22, 3-5. Poiché la glossa apparentemente 'ritmica' di Esichio non presenta il termine $\beta \alpha \chi \chi \varepsilon \tilde{i o s}$ per identificare il coriambo, sospetterei in alternativa che si faccia riferimento ad una mixtio di metra giambici e trocaici.

${ }^{46} \mathrm{La}$ notizia è confermata dallo scolio a questo passo: cf. schol. ad Pl. Lg. 800 e p. 329 Greene. Le prefiche carie

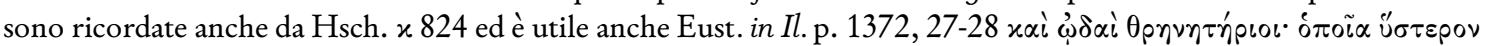

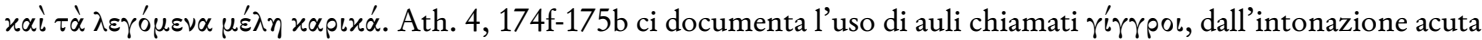
e lamentosa, di cui "si servivano anche le genti della Caria nei lamenti funebri", per cui $c f$. anche Poll. 4, 76 che lo

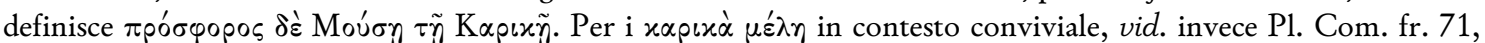
12-13 Kassel-Austin (= Ath.15, 665b).

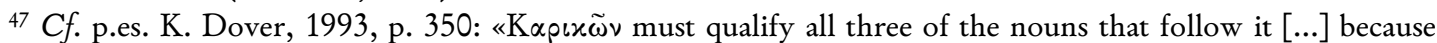

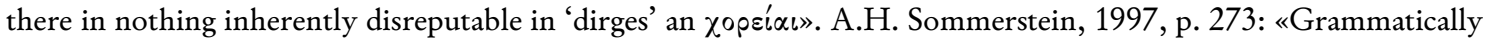
the Greek does not it clear whether "from Caria" applies to all three nouns or only to the first ("pipe-tunes"), but sense requires that it apply to all three since there in nothing untragic about dirges (thrēnoi) as such». Chi voglia

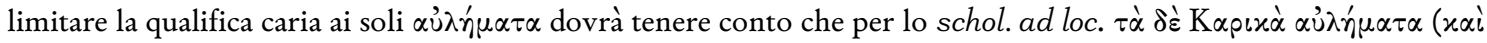

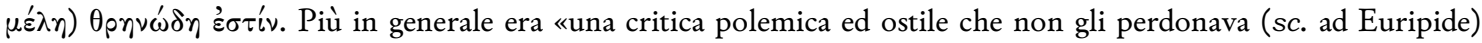
l'audacia di aver riempito i suoi drammi di arie popolari, lamentevoli ed esotiche che avevano il solo scopo di coinvolgere emozionalmente il pubblico»: G. Comotti, 2017, p. 36. La considerazione della melodia caria era negativa, anche perché proveniente da una regione da cui giungeva ad Atene mano d'opera servile e prezzolata: $c f$. p.es. schol. rec. ad Ar. Ra. 1302c, p. 217, 14-17 Chantry. Resta utile il più tardo giudizio moraleggiante di Clem.

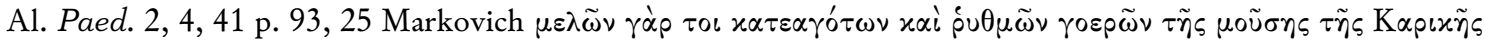

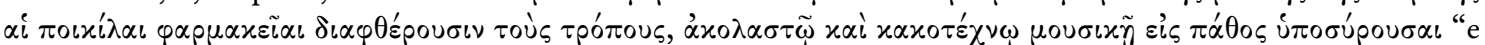
infatti i variegati incantesimi delle effeminate melodie e dei lugubri ritmi della musa caria corrompono le indoli, e con una musica dissoluta e depravata inducono al turbamento della mente”. 
Nell'epodo, concentrato sul destino dei figli di Eracle, i due ipodocmi iniziali (vv. 131 e 132) equivalenti a pentemimere trocaici, ${ }^{48}$ staccano verso un tono più trepidante, ${ }^{49}$ perché l'attenzione dei coreuti da se stessi e dalla propria condizione si è spostata ora verso i piccoli figli di Eracle.

Le edizioni moderne modificano in più punti la colometria manoscritta, allo scopo di uniformare i cola all'andamento giambico nella diade antistrofica ${ }^{50}$ e all'andamento trocaico nell'epodo, annullando questa commistione di cola giambici e trocaici, che invece ha una sua ratio ed è una cifra ritmica fondante. Le modifiche più ricorrenti sono l'introduzione della sinafia verbale

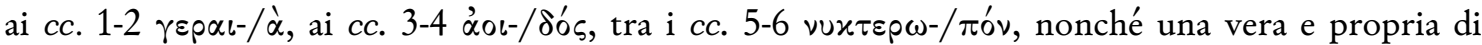
riscrittura colometrica dei cc. 9-10. Nell'epodo è frequente l'introduzione della sinafia verbale tra i cc. 1-2 $\gamma \circ \rho-/ \gamma \tilde{\omega} \pi \varepsilon \varsigma$. Nessuna di queste modifiche appare necessaria né consigliabile, anzi contrasta, dal punto di vista metrico-ritmico, con lo stile euripideo nel genere del lamento funebre orientaleggiante..$^{51}$

\section{Tradizione e critica del testo}

v.107 119. Sul testo e sulla responsione rinvio a quanto argomentato in altra sede. ${ }^{52}$ Questo colon rappresenta semplicemente una misura più breve di quello del v.111 124.

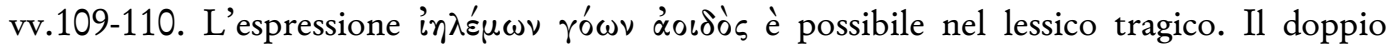
genitivo in asindeto è parso infastidire perché la parola íñ $\varepsilon \varepsilon \varsigma_{s}$ è attestata nel greco classico solo come sostantivo, ${ }^{53} \mathrm{ma}$ la iunctura si può spiegare come un caso di specie particolare accostata ad un nome generico, nell'àmbito dei termini relativi alla lamentazione. Il linguaggio tragico tende all'asindeto, più spesso con verbi vicini tra loro per significato, talora tra aggettivi e nomi di significato affine: $c f$. p.es. E. Hec. $70 \delta \varepsilon^{\prime} i \mu \alpha \sigma \iota \varphi^{\prime} \alpha \sigma \mu \alpha \sigma \iota v{ }^{54}$ Eे possibile conservare il testo tràdito anche in base ad un uso aggettivale del sostantivo. Essendo due sostantivi accostati, uno può essere avvertito come aggettivo: $:^{55}$ in questo caso si intenderà p.es. "aedo di lugubri lamenti”. È inutile la normalizzazione 'dorica' ' $\alpha \lambda \varepsilon$ ' $\mu \omega \nu$ : per il vocalismo 'ionico' tràdito, cf. E. Supp. 281, A. Supp. 115, A. Ch. 424 in $\lambda \varepsilon \mu \iota \sigma \tau \rho i \alpha \varsigma .{ }^{56}$

${ }^{48}$ I due schemi vanno interpretati tenendo conto l'uno dell'altro, $c f$. M. West, 1982, p. 110 n. 92: «If the first position is resolved there is nothing to distinguish $h d$ to $d$ ». L'ipodocmio con chiusa 'pesante' qui sembra piuttosto affine all'andamento trocaico. Per questo schema, in contesti non docmiaci, cf. p.es. E. Heracl. 898 in M. G. Fileni, 2006, p. 80; E. Rh. 528 in G. Pace, 2001, p. 45: «in questo contesto la sequenza è forse sentita come una forma ampliata di epitrito trocaico».

${ }^{49}$ Lo confermano anche l'anadiplosi di ő́ous al v. 136 e l'inversione 'E $\lambda \lambda \dot{\alpha} \varsigma \widetilde{\omega}$ al v. 135.

${ }^{50}$ Riassume bene la communis opinio A. M. Dale, 1983, p. 237: «107ff. pure iambic with much resolution and long pnigos in last period 113-6, 125-8».

51 Tanto è vero che «tipica dello stile metrico di Euripide è l'associazione asinarteta 2ia / ithyph [...] al punto che il verso fu chiamato “euripideo”»: B. Gentili \& L. Lomiento, 2003, p. 128.

52 P. Santé, 2019, pp. 133-138.

${ }^{53}$ Per l'uso come aggettivo, ma con il significato differente e successivo di “noioso" e "sciocco", $c f$. p.es. Luc. Pseudol. 24; Gal. 14, 617 Kühn. Una più articolata gamma sinonimica è in Hsch. ı 28 e 30 Latte.

54 Più ampiamente vid. R. Rehenan, 1985, pp. 147-149 e M. S. Mirto, 1997, pp. 106-107, n. 13. Per l'accumulo di più termini riferibili all'àmbito della lamentazione, cf. E. Hec. 297-298, Cyc. 69-70, El. 141-144. La minima

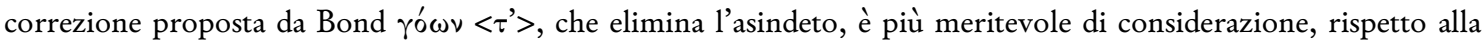

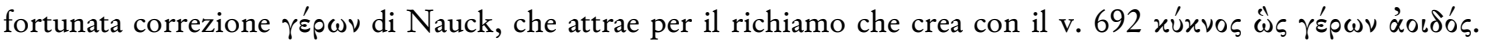
L'asindeto può avere la funzione stilistica di assommare, quasi accumulare, più rapidamente i canti di lamento: sul suo uso vid. Demetr. Eloc. 4, 194 e Longin. Subl. 19.

55 Vid. p.es. W. J. Verdenius, 1987, p. 7 e di rimando p. 2: «Euripides seems to have a predilection for the

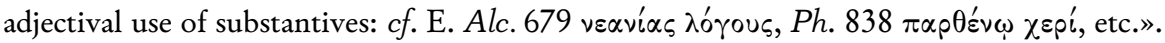

56 L'alternanza tra in $\lambda \varepsilon \mu \circ \varsigma$ e ía $\lambda \eta \mu \circ \varsigma$, nei drammi di Euripide, va probabilmente ricondotta alla vicende della trasmissione, in quanto le tragedie 'alfabetiche' presentano la forma iń $\lambda$-, quelle con scoli la forma i $\alpha \lambda-$. Secondo G. Björck, 1950, p. 161 la forma i̊́ $\lambda \eta \mu o s$ sarebbe secondaria. 
Paolo Santé

La parodo dell'Eracle di Euripide: tradizione critica della colometria e del testo

v.111. La lezione ơvo $\mu \alpha$ di tradizione indiretta tramite Plu. De comm. not. 1066c è metricamente equivalente all' $\varepsilon \kappa \varepsilon \alpha$ della tradizione diretta, ma il contesto è un'evidente parodia in

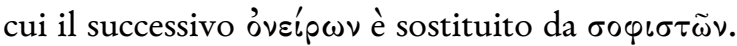

v.113. Il tràdito $\mu^{\prime}$ vov va corretto in $\mu \varepsilon^{\prime}$ : non può essere accettato perché introduce la

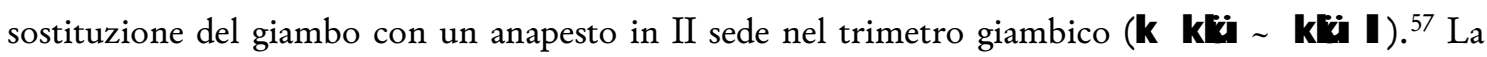
congettura di Tyrwitth va accolta. Viceversa il tràdito $\pi \rho o ́ \theta u \mu \alpha$ non deve essere modificato in $\pi \rho o ́ \theta u \mu ’$ seguendo Elmsley, perché la misura ipercatalettica del dimetro giambico dà la clausola finale, in coincidenza con lo iato e la pausa di senso. ${ }^{58} \mathrm{Ne}$ consegue che non vada corretto in $\pi \alpha \rho \alpha x^{\prime} \mu_{i \iota} \zeta^{\prime}$ il $\pi \propto \rho \alpha x^{\prime} \mu \iota \zeta \varepsilon$ del v. 126 dell'antistrofe: la necessità della duplice elisione è estremamente sospetta. ${ }^{59}$ Sintatticamente le parole $\tau \rho \circ \mu \varepsilon \rho \alpha ́$ e $\pi \rho o ́ \theta u \mu \alpha$ sono ambigue: possono riferirsi all'’' $\pi \varepsilon \alpha$ del v. 111, ma

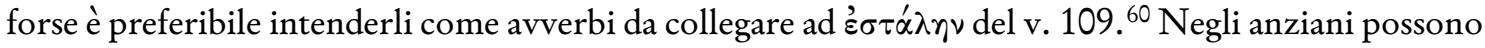
essere 'tremolanti' sia le parole, sia i corpi in movimento.

v.114/115 127. Con il testo dei codici il verso è un trimetro giambico, in cui si possono riconoscere alcune particolarità prosodiche: i⿳亠丷 monosillabico per sinizesi, che permetterebbe di non ricorrere alla correzione $\widetilde{\omega}$ di Hermann, ${ }^{61} \pi \alpha \tau^{\prime}$ pòs con allungamento davanti a muta cum liquida e $\gamma \varepsilon p \alpha \iota \iota^{\prime}$ con abbreviamento in iato. ${ }^{62}$ La correzione $\tilde{\omega}$ di Hermann resta preferibile per il parallelismo con il successivo vocativo, ma sarebbe possibile mantenere l'esclamativo i $\omega$ che sottolineerebbe l'amarezza alla vista dei ragazzini, cui si associano l'enfasi nella ripetizione del genitivo di separazione

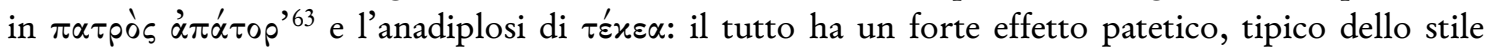
euripideo, all'interno di un trimetro giambico quasi interamente soluto. ${ }^{64} \mathrm{E}$ possibile una scansione

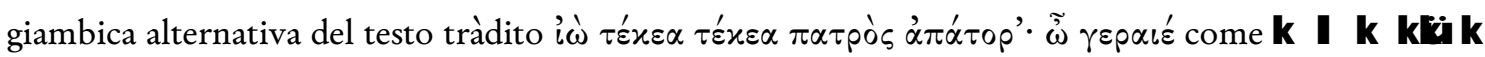

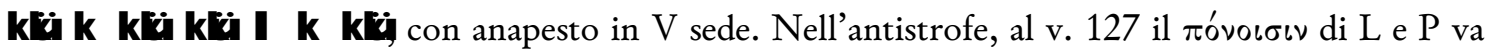
conservato, sebbene sia stato modificato da Triclinio in tóvoıs per inserire uno schema giambico (vid. supra) e tale modifica sia stata generalmente accolta nelle edizioni moderne.

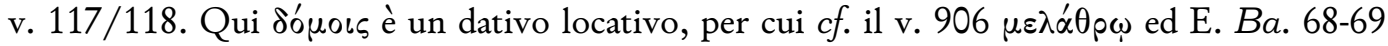

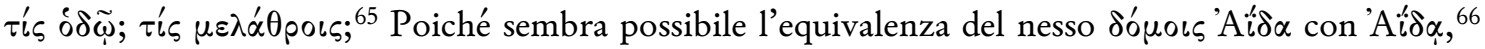

${ }^{57} \mathrm{La}$ sostituzione anapestica in sede pari è un'eventualità estremamente rara nel trimetro giambico della tragedia: cf. B. Gentili \& L. Lomiento, 2003, p. 131 e p. 258.

${ }^{58}$ Cf. B. Gentili \& L. Lomiento, 2003, p. 136. Per il 2ia hypercat, cf. S. Tr. 102 111, in un contesto di dattiloepitriti; in contesti giambici e trocaici cf. p.es. E. Rh. 828 in G. Pace, 2001, p. 56, ia + reiz nell'analisi di Pace; S. OC 1691, ia + penthem ${ }^{i a}$ nell'analisi di L. Lomiento, 2008, p. 398; S. El. 484 500, per cui cf. L. Lomiento, 2019, p. 372; Ar. Lys. 1316, 1318 e 1320 in F. Perusino, 2016, p. 88, ia + reiz nell'analisi di Perusino.

${ }^{59}$ Nell'antistrofe per ottenere l'elisione è necessaria anche la traiectio tra i vv. 126 e 127 proposta da Musgrave, che altera l'ordine dei versi: vid. oltre. Sia $\pi \rho o ́ \theta u \mu \alpha$ nella strofe che $\pi \alpha \rho \alpha x^{\prime} \mu_{\iota} \zeta \varepsilon$ nell'antistrofe sono conservati p.es. da L. Dindorf, 1825 e F.A. Paley, 1880².

$60 \mathrm{Il}$ neutro plurale degli aggettivi è usato come avverbio, particolarmente con i verbi di movimento: $c f$. p.es. S.

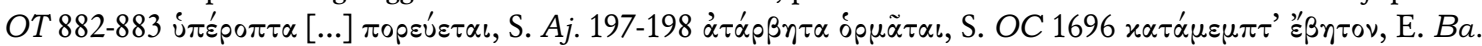

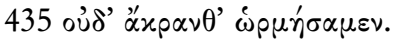

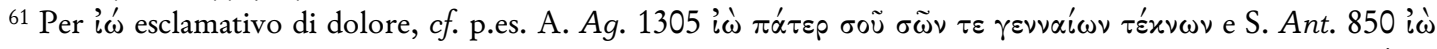

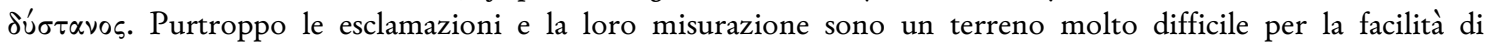
corruzione: le correzioni moderne sono estremamente frequenti, giuste o meno che esse siano.

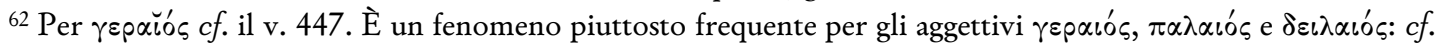
J. Diggle, 1974, p. 15 n. 7, G. W. Bond, 1981, p. 97, M. West, 1982, p. 11.

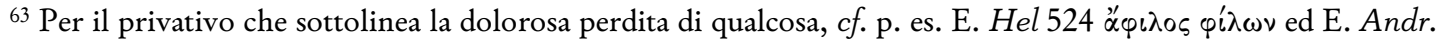

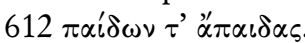

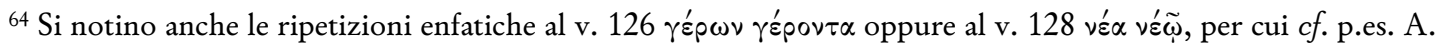
Pr. 955, S. Ant. 1266, OC 1448, E. Ion 713.

65 Secondo la punteggiatura di L e P.

66 Per l'assenza della preposizione, vid. M. Sanz Morales, 1988, p. 308 che fa notare, in base al confronto tra

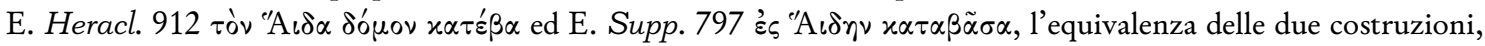
indipendentemente dalla presenza della preposizione di movimento; contra vid. le osservazioni di J. Diggle, 1981, 
Paolo Santé

La parodo dell'Eracle di Euripide: tradizione critica della colometria e del testo

ne consegue che l'integrazione di $\langle\dot{\varepsilon} v\rangle$ davanti a $\delta^{\prime}$ 'roıs, proposta da Hermann e largamente accolta nelle edizioni moderne, non sia necessaria per ragioni linguistiche. ${ }^{67}$ Vista la presenza del genitivo tipicamente 'omerico' $\tau \rho \circ \chi \eta \lambda \alpha^{\alpha} \tau 0 \iota 0$ al v. 123 , sospetto che, integrando la preposizione, venga anzi rimossa una voluta patina linguistica arcaicizzante. ${ }^{68} \mathrm{La}$ misurazione 'A $\overline{\mathrm{A}} \mathrm{i} \delta \alpha$ è meno frequente ma pienamente ammissibile: $c f$. p.es. E. El. 122 e 144; E. fr. 936 Kannicht; S. OC 1690.69

vv. 121/122-123. Questi versi presentano un coacervo di difficoltà sintattiche, semantiche e metriche, che ha generato un numero esorbitante di congetture, nessuna delle quali si è imposta sulle altre. ${ }^{70}$ Infatti il passaggio, in più recenti e autorevoli edizioni, si presenta con la crux desperationis: G. Murray, 1913³, app. ad loc.: «varie tentati»; L. Parmentier \& H. Grégoire, 1923, app. ad loc.: «121 sq. corruptum»; J. Diggle, 1981; K.H. Lee, 1988, app. ad loc.: «locus conclamatus».

Dal punto di vista della sintassi, la prima difficoltà è che una costruzione di $\omega \sigma \tau \varepsilon$ con il

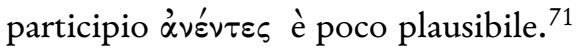

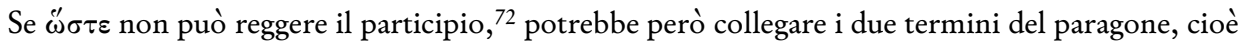
il piede e la gamba pesante con il puledro aggiogato. Un'ipotesi è che il verbo $\pi \rho \circ x \alpha ́ \mu \eta \tau \varepsilon$ (v. 119) regga il participio $\alpha^{\prime} \nu \varepsilon^{\prime} v \tau \varepsilon \varsigma$ (v. 121a) in funzione predicativa e con forte iperbato. ${ }^{73}$ Un'altra

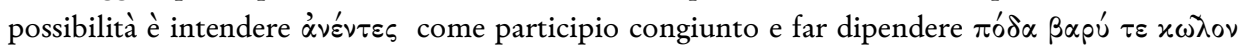

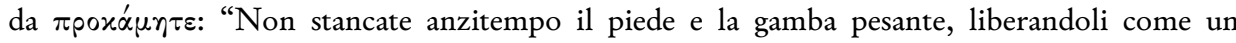
puledro aggiogato lungo una salita ripida". Ci sarebbe una forma di comparatio compendiaria, equivalente a "liberandoli come i piedi e le gambe di un puledro aggiogato". ${ }^{74}$

p. 47 per il quale, solo davanti ai nomi propri verrebbe omessa la preposizione, con i relativi esempi: S. OT 899, E. Heracl. 360-361, E. Hipp. 545-546, E. IT 156, E. Tr. 171-172.

${ }^{67} \mathrm{Cf}$. H. White, 2000, p. 58. Per il locativo senza preposizione, oltre ai casi già citati, cf. p.es. S. OT 817 dónoıs

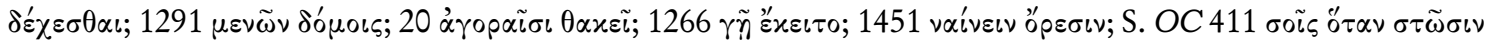

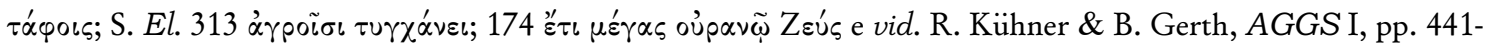
442.

${ }^{68}$ Cf. O. Hoffmann \& A. Debrunner, GGS, pp. 110-111: «Die klassische Sprache geht auf dem Weg weiter: während Homer noch den lokativischen Dativ ohne Präposition brauchen kann [...] ist das im Attischen nur noch erstarrt in Ortsnamen möglich [...]»; J. Humbert, 1945, p. 292: «On peut mesurer l'importance du recul qu'a subi le datif locatif spatial, entre Homère et l'attique, quand on voit avec quelle liberté le datif locatif sans préposition est employé dans l'épopée».

${ }^{69}$ Nonché Semon. fr. 1, 14 e fr. 7, 17; Herod. 3, 17. Per la misurazione lunga, cf. anche 'Āítos in Hom. Il. 6, 284.

70 Un accurato elenco in N. Wecklein, 1899, p. 57 e poi in C. Neri, 2003, p. 71. In certi casi il testo emendato

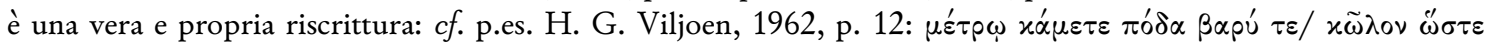

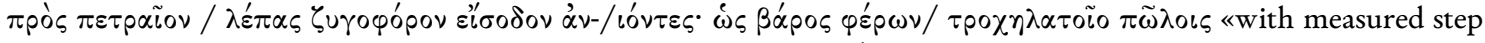

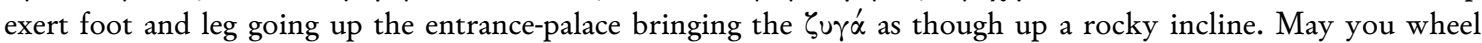
along like a horse drawing a load».

71 Per $\dddot{\omega} \sigma \tau \varepsilon$ con il participio ci sono alcune occorrenze in Erodoto e due in Tucidide (2, 40, 4 e 7, 24, 2), per cui vid. p.es. J. D. Denniston, GP, pp. 526-527 e p. 588, nonché R. Kühner \& B. Gerth, AGGS II, p. 97: «Häufig ist es bei Herodot, höchst zweifelhaft bei den Attikern». In A. Ag. 884 il tradito $ڤ \sigma \tau \varepsilon$ con participio sottinteso è corretto con $\dddot{\omega}_{\varsigma} \tau \iota$ di Hartung: $c f$. da ultimo E. Medda, 2017, p. 50.

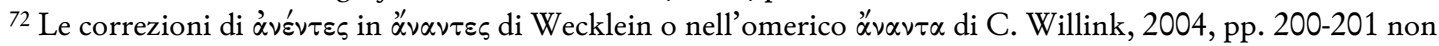

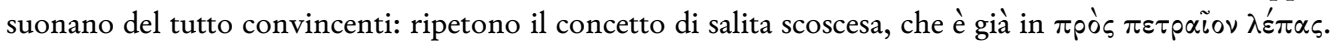

${ }^{73}$ Lo si legge in C. Neri, 2003, p. 73, n. 22 in cui si precisa «che è emersa nel corso di un seminario con Renzo Tosi» per poi tradurre: «Non sovraffaticatevi nel far salire i piedi e le pesanti gambe [...]». Quella di $x \alpha ́ \alpha \nu \omega$ con gerundio è una costruzione ampiamente attestata: cf. p.es. A. Eu. 881, E. IA 1143, Or. 1590, Ar. Lys. 541/542.

${ }^{74}$ Le clausole comparative sono suscettibili di varie abbreviazioni o intrecci con la principale: vid. R. Kühner \& B. Gerth, AGGS II, p. 492 e per un caso simile, $c f$. Hipp. Morb. Sacr. 1, 85 in cui " $i \pi \omega$ è brachilogia per

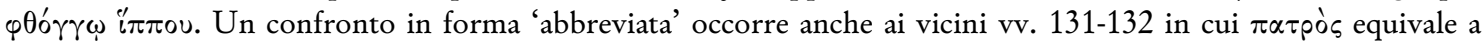

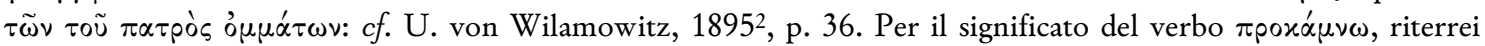
preferibile quello di "sfinirsi anzitempo", cioè prima di aver raggiunto la propria meta, per cui $c f$. Thgn. 925 e A. $E u .78$, come già sosteneva p.es. J.W. Verdenius, 1987, p. 7: «do not weary in your foot before you have reached your destination». Per il significato del verbo ávín $\mu \iota$, riterrei consigliabile quello di "lasciare andare a briglie sciolte", 
Paolo Santé

La parodo dell'Eracle di Euripide: tradizione critica della colometria e del testo

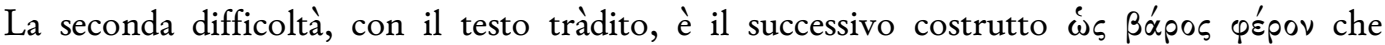
apparentemente è un accusativo assoluto con verbo non impersonale. ${ }^{75}$

Andrebbe reso con un costrutto in cui il sostantivo fa da soggetto e il participio da predicato: il che è poco perspicuo. ${ }^{76}$ Tuttavia il sostantivo che funge da soggetto del participio può essere omesso, se si aggiunge facilmente dal contesto. ${ }^{77} \mathrm{Si}$ potrebbe sospettare che il logico soggetto del verbo sia stato sottinteso e sia dunque la gamba (o il piede) in quanto è essa che porta il peso nel movimento, sia del corpo anziano (se l'arto è umano), sia del carro a cui è aggiogato il cavallo

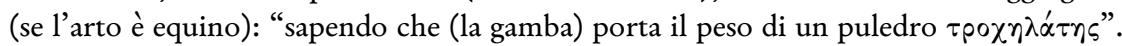

Dal punto di vista semantico ci sono ulteriori difficoltà: proprio il successivo nesso $\tau \rho \circ \chi \eta \lambda \alpha^{\prime} \tau \circ \iota 0 \pi \omega^{\prime} \lambda$ ou non è di facile interpretazione.

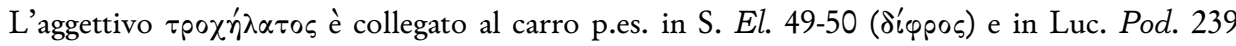
$\left(\alpha \pi \eta^{\prime} \sim \eta\right)$ dove va inteso come "trainato da ruote", cosa ovvia per un carro. Ma il senso passivo qui è impossibile, in quanto il puledro "non è trainato", sia mai "traina”, per cui sarà un genitivo con desinenza epica, che non va ricondotto all'aggettivo $\tau \rho \circ \chi \eta^{\prime} \lambda \alpha \tau \circ \varsigma$ "trainato su ruote", bensì

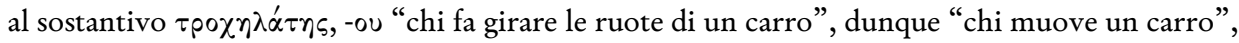
definendo un cavallo da tiro o che comunque traini un carro. ${ }^{78} \mathrm{Di}$ una certa utilità potrebbe essere S. E. Adv. Math. 9, 228-229 che, nell'àmbito di un ragionamento sui principi di causa ed

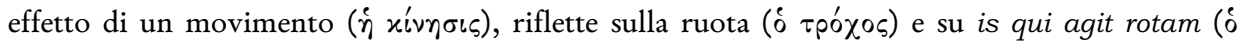
$\left.\tau \rho \circ \chi \eta \lambda \alpha^{\prime} \eta \xi\right)$. Se si parla delle ruote di un carro, è il cavallo che genera il movimento, sia autonomamente sia come conseguenza dello sprone del carrettiere. ${ }^{79}$ Se il corpo anziano è un pesante fardello, cioè un carro da trasportare, allora i vecchi sono cavalli da tiro perché trainano

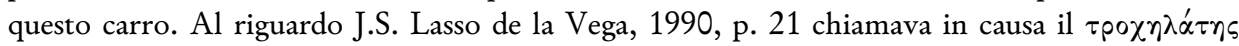

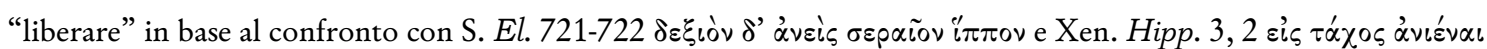

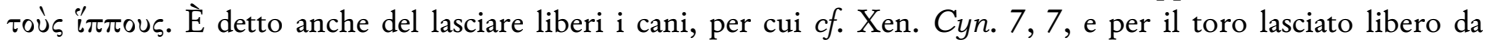

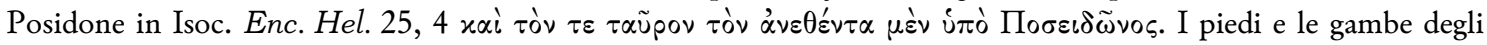
anziani dal passo troppo precipitoso per l'età sarebbero come un puledro aggiogato a briglie sciolte lungo una salita ripida. Non mi risulta segnalato da alcuno che la similitudine tra gli arti umani e un cavallo in movimento sia reperibile almeno in un altro luogo. È un passo delle Eroidi di Ovidio 18, 161-6 in cui le braccia di Leandro che nuota in mare, sono paragonate a un cavallo in corsa: saepe per adsiduos languent mea bracchia motus,/ vixque per immensas fessa trahuntur aquas./ his ego cum dixi: "pretium non vile laboris, / iam dominae vobis colla tenenda dabo", / protinus illa valent atque ad sua praemia tendunt,/ ut celer Eleo carcere missus equus. Per il paragone con il cavallo spossato in salita, due noti loci similes sono Petron. Satyr. 134, 2 mollis, debilis, lassus tamquam caballus in clivio e Ov. Rem. Am. 394 principio clivi noster anhelat caballus.

${ }^{75} C f$. M. Lacroix, 1967, p. 141, in cui però la proposta di correzione di $\pi \tilde{\omega} \lambda$ ov in $\pi \hat{\lambda} \lambda$ ov, attribuita a J.P. Mahé, oltre che semanticamente per me poco convincente («laissant partir la roue qui porte l'attelage») fa difficoltà alla

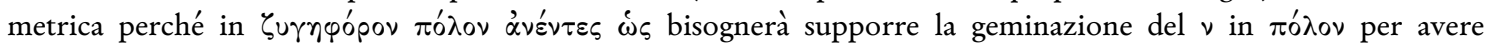
l'andamento giambico.

${ }^{76}$ Infatti generalmente è preferita la causale con $\omega$ 's e participio o ottativo, che comporta la correzione del tràdito

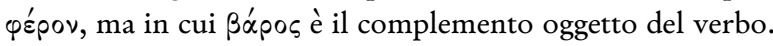

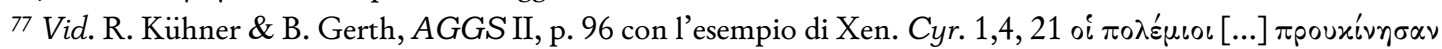

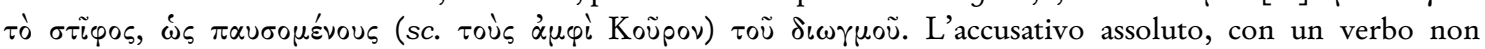
impersonale introdotto da $\mathfrak{\omega}_{\zeta}$, è un costrutto attestato nella lingua tragica: $c f$. p.es. S. OT 101; S. El. 882; S. Ph. 1065; E. Ion 965; E. Ph. 1461 e 714; E. Rh. 145; E. Heracl. 693.

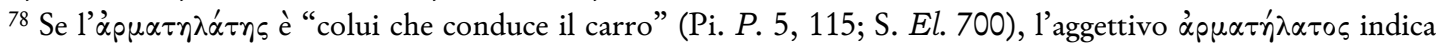

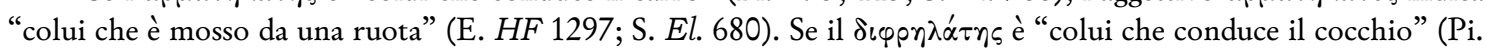

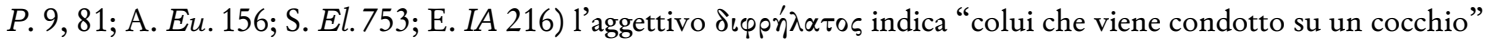
(E. fr. 1108 Nauck $^{2}=R h$. arg. b). Se l'i $i \pi \eta \lambda \lambda^{\alpha} \tau \eta$ è "colui che cavalca un cavallo" (Hom. Il. 4, 387; E. Rh. 117), l'aggettivo i $\pi \pi \dot{n} \lambda \alpha \tau o \varsigma$ vuol dire "percorribile a cavallo" (Hom. Od. 4, 607; 13, 242).

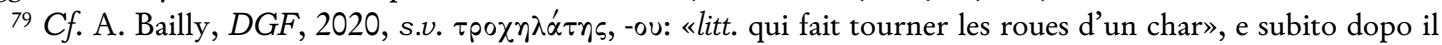
significato «cheval attele»» per S.E. Adv. Math. 9, 228-229. Diversamente, come aggettivo lo intende qui LSJ s.v. $\tau \rho \circ \chi \hat{n} \lambda \alpha \tau \circ \varsigma$, -ov: «metaph., hurried along like a wheel or a chariot E. HF 122». 
"i $\pi \circ \varsigma$ equivalente al curulis equus, secondo alcuni glossari greco-latini. ${ }^{80} \mathrm{Ad}$ ogni modo, di fatto, il termine è attestato solo per indicare il carrettiere: cf. S. OT 806, E. Ph. 39 ed E. Rh. 950 dove è correzione di Valckenaer per $\sigma \tau \rho \alpha \tau \eta \lambda \alpha^{\prime} \tau \eta \varsigma^{81}$

Un ulteriore problema è che il termine puledro si ripete a breve distanza due volte ( $\pi \omega \lambda$ ov ... $\left.\pi \omega^{\prime} \lambda \circ \mathrm{u}\right)$ in maniera sospetta, ${ }^{82}$ per cui molti studiosi hanno proposto l'inserzione dei più svariati mezzi di trasporto al posto di una delle due occorrenze. ${ }^{83}$

A mio modo di vedere, la scelta del puledro $(\pi \tilde{\omega} \lambda \circ \varsigma)$ al posto del cavallo (i $i \pi \pi \circ \varsigma)$, termini metricamente equivalenti, ha un senso: del giovane puledro i vecchi non hanno le forze, ma hanno lo spirito, l'impeto 'senza freni' con cui sopraggiungono, perché arrivano con il passo dei giovani, ma non lo possono certo reggere, per il loro fisico senescente. ${ }^{84}$ Nella scelta del $\pi \omega \lambda_{0 \varsigma}$ si può avvertire anche un gioco fonico con il termine "gamba" $(\varkappa \tilde{\omega} \lambda \circ \nu)$. Ne deduco che eliminare per congettura entrambe le occorrenze, come fanno p.es. M. West, 1973, pp. 145-151 o C.

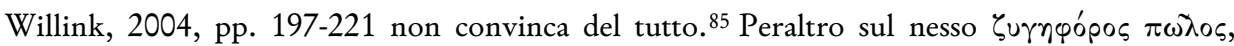
tranne che per le ragioni metriche di cui dirò, non sembra lecito dubitare: è attestato in A. fr.

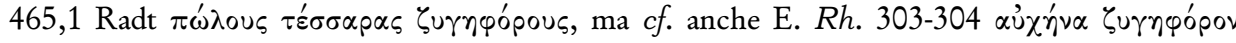

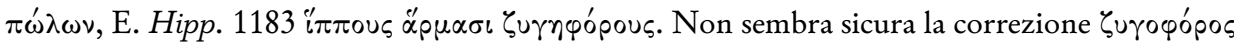
perché è una forma prosastica e successiva: vid. Plu. De cup. div. 2, 524a.

Dal punto di vista metrico il $\pi \tilde{\omega} \lambda$ ov del v. 121/122 introduce un'anomalia difficilmente spiegabile come responsione libera, almeno secondo la colometria manoscritta. Si tratta dell'unico punto in cui lo schema metrico dell'antistrofe $\left(\begin{array}{l}k\end{array}\right)$ non ripete quello della strofe $(k \mid) .{ }^{86}$

${ }^{80}$ La sua proposta è seguita da Calderón Dorda, 2002, p. [6]. Il termine curulis, corrispondente a currulis, di cui è variante grafica o di pronuncia tendente a confondersi nella tradizione manoscritta, è usato per il cavallo che traina un carro (currus). Il termine cu(r) rulis, se collegato al cavallo, è un'accezione tecnica per i cavalli che gareggiano in una corsa di carri, di cui c'è traccia nell'uso del tardo impero, per cui vid. p.es. E. Rawson, 1981, 116 (spec. pp. 5-6) e J. A. Jiménez Sanchéz, 2012, pp. $481-490$ (spec. p. 482, n. 2). Infatti nei glossari greco-latini si

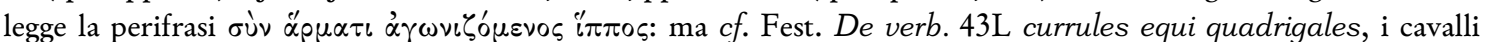
forniti per trainare carri o quadrighe in sfilate solenni o ai giochi nei circhi. La discrasia temporale resta ragguardevole: tuttavia vid. p.es. J. J. Reiske, 1753, p. 162: «123 vet. ed. $\pi \omega \dot{\lambda}$ ou, unde efficio $\beta \alpha \rho u \sigma \varphi u ́ p o u$ $\tau \rho \circ \chi \eta \lambda \alpha^{\prime} \tau 0 \iota 0 \pi \dot{\omega} \lambda$ ००, equi currum trahentis, cui onere cursus et longo itinere pes gravis et tardus factus est».

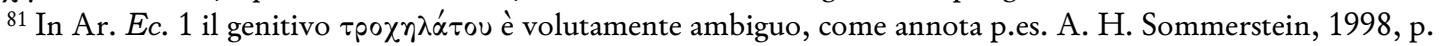
137: "Greek trokhēlatou (genitive case), which could either from trokhēlatēs "driver on wheels, charioteer" (appropriate to the Sun, cf. Clouds 571-574, S. Aj. 851, etc.) or from trokhelatos "driven/propelled on whells/a whell” (appropriate to a product of the potters art, cf. 4)».

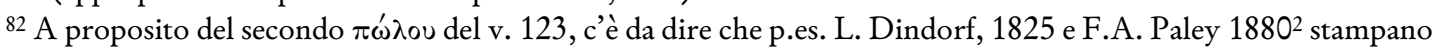

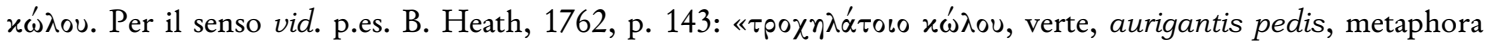
ducta ab aurigatione, ut saepe alias a remigatione». Sebbene la confusione tra $\pi \dot{\omega} \lambda$ ov e $\chi \omega \dot{\omega} \lambda$ ov sia molto facile, non compare in alcun recentior e il solo Laur. 31,1 ha l'errato $\pi$ ónou.

${ }^{83}$ Esprime bene la communis opinio C. Neri, 2003, p. 72: «ciò che pare certo è che o $\pi \tilde{\omega} \lambda$ ov (v.121) o $\pi \dot{\omega} \lambda$ ov

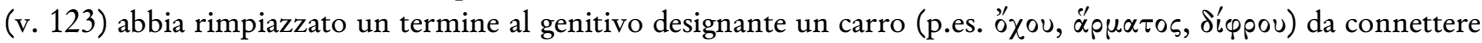

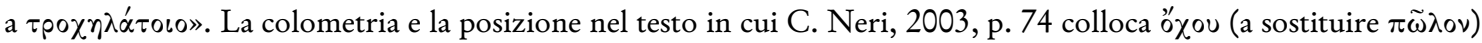

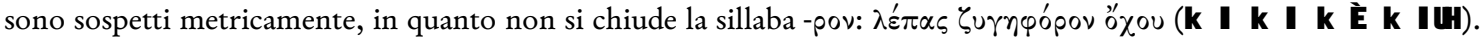

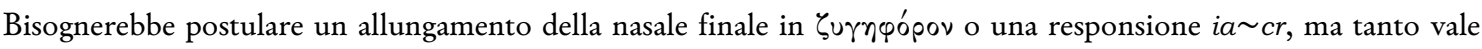
risolvere con il d'́ppou di J. Baumann, 1862 citato in apparato. Non senza una punta di sarcasmo, C. Willink, 2004,

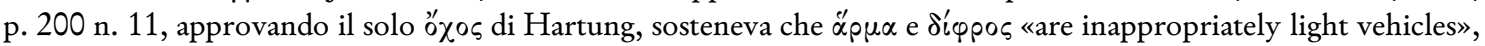

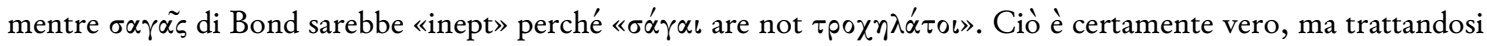
di un percorso in salita, non direi così necessario che il veicolo trainato debba essere anche molto pesante.

${ }^{84}$ Ciò risponde all'obiezione di G.W. Bond, 1981, p. 98 circa il testo tràdito, secondo il quale «the urging on of an horse $[\ldots]$ is not properly parallel to the chorus' personal effort».

${ }^{85}$ Cf. G.W. Bond, 1981, p. 98: «The elimination of both instances of $\pi \tilde{\omega} \lambda \circ \varsigma$ seems unduly drastic».

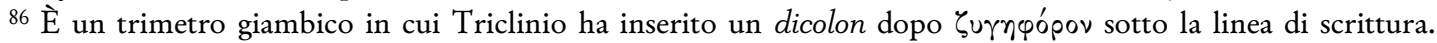
Dunque anche lui aveva notato una 'difficoltà' in questo punto dello schema: vid. supra. 
Paolo Santé

La parodo dell'Eracle di Euripide: tradizione critica della colometria e del testo

Si potrebbe rendere conto delle differenze tra i due schemi postulando nel secondo metron una responsione ba ia $(k|k| \sim k||)^{87}$ cui si aggiungerebbe un'alogos giambica soluta nel terzo metron ( $k|k| \sim k|k| k \mid)$ secondo la nota possibilità della sostituzione anapestica del primo piede del giambo: $c f$. Heph. Ench. p. 15, 17-20. La coesistenza dei due fenomeni desta

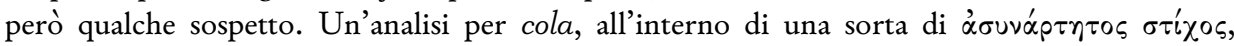
comporterebbe due possibilità: $2 i a \mu+2 i a \mu(\sim 2 c h o \mu)$ oppure penthem ${ }^{i a}+2 \operatorname{tr} \wedge\left(\sim\right.$ ion $^{\text {mai }}$ tr $\left.\wedge\right)$. La prima è preferibile perché l'anaclasi del giambo $(k|k|)$ e del coriambo $(|k k|)$ è già al c. 1. Si tratta di metra affini, come il trocheo $(k-k \mid k)$ con lo ionico, tanto a minore ( $k$ | | ) quanto a a maiore (l | k k ). ${ }^{88}$ Resta il fatto che non sembra un 'falso' trimetro giambico, nato dall'accorpamento di cola autonomi, ${ }^{89}$ vista la corrispondenza tra la colometria manoscritta della strofe e quella dell'antistrofe. Ciò mi induce a non intervenire con una modifica della colometria, che permetterebbe di inserire l'anomalia metrica in una plausibile responsione libera, p.es. come segue:

\begin{tabular}{|c|c|c|}
\hline$\lambda \varepsilon^{\prime} \pi \alpha \varsigma \zeta u \gamma \eta \varphi o ́ p o \nu$ & k l $\quad k \quad k \quad k \quad$ l & $2 i a^{\wedge}$ \\
\hline 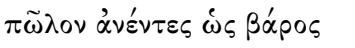 & $\begin{array}{llllllll}a & u & k & k & k & k & k\end{array}$ & $2 i a(\sim \operatorname{cho} i a)$ \\
\hline$\varphi \varepsilon^{\prime} \rho \circ \nu \tau \rho \circ \chi \eta \lambda \alpha^{\prime} \tau 0 \iota 0 \pi \omega^{\prime} \lambda \circ \nu$ & $\begin{array}{llllllll} & \mid & k & k & k & k & k\end{array}$ & 2ia hypercat ${ }^{90}$ \\
\hline
\end{tabular}

Alla luce di un quadro piuttosto dubbio sotto molteplici punti di vista sintattici, semantici e metrici, anche io tenderei a concludere che il testo tràdito dai manoscritti sia in qualche misura corrotto.

vv. 126-127. Non è necessaria la traiectio tra i due versi proposta da Musgrave e largamente accettata nelle edizioni più recenti: per l'iperbato che pospone il relativo, vid. p.es. S. Tr. 200 tòv

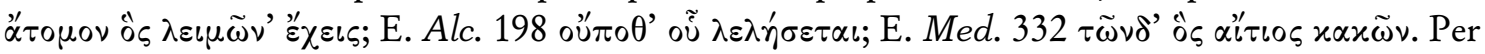

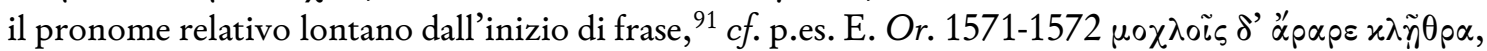

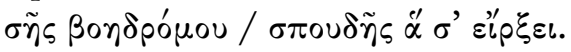

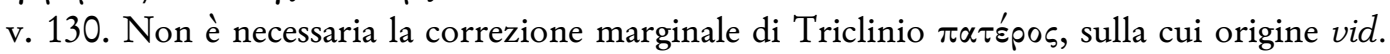
supra. La si può accettare, se si vuole evitare l'allungamento in $\pi \alpha \tau^{\prime}$ pós: ${ }^{92}$ con $\pi \alpha \tau$ ع́pos si avrebbe un hypodo (= penthem $\left.{ }^{t r}\right)$ di schemak k k k k k l.

\section{Bibliografia}

Edizioni e/o commenti citati con eventuale traduzione dell'Eracle ${ }^{93}$

S. Barlow, 1996, Euripides' Heracles, Warminster.

G. W. Bond, 1981, Euripides. Heracles, Oxford.

${ }^{87} \mathrm{La}$ responsione si risolverebbe con un 'superallugamento' del baccheo: si vedano i due cretici del v. 135 (c. 6 dell'epodo) estranei al contesto metrico giambo-trocaico, possibili cretici 'superallungati'. Per questo genere di responsioni, vid. B. Gentili \& L. Lomiento, 2003, pp. 221-223.

${ }^{88}$ In questa direzione testimonia il frammento di Heph. Ench. p. 77, 10-11 (= schol. in Hermog. Rhet. Gr. 7,

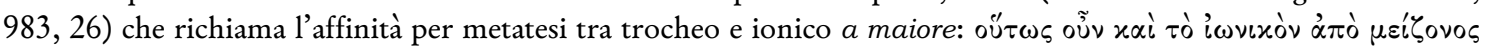

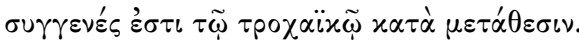

${ }^{89}$ Come invece interpreta Triclinio con il suo dicolon. La fusione di cola autonomi su una stessa linea è una comune forma di corruzione della colometria. Qui sarebbe avvenuta parallelamente nella strofe e nell'antistrofe, un'eventualità che lascia scettici.

90 Riporto per brevità il testo della sola antistrofe; nella strofe ci sarebbe una sinafia verbale. Il 2ia^ (= pros $\left.{ }^{d}\right)$ è una comune misura giambica brachicatalettica e la responsione tra ia e cho è tra le meglio attestate ed è già al c. 1: per altre sue attestazioni nella prassi teatrale e per la sua spiegazione dal punto di vista teorico, rinvio a quanto già argomentato in P. Santé, 2019, pp. 133-138. Il 2ia hypercat ritornerebbe al c.7.

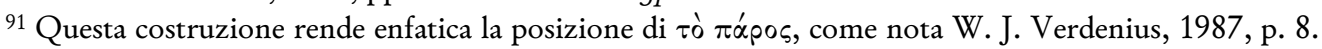

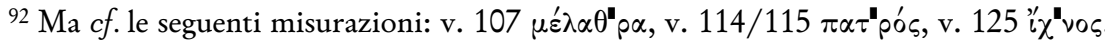

${ }^{93}$ L'elenco si limitata alle sole edizioni e commenti citati o più recenti. 
Paolo Santé

La parodo dell'Eracle di Euripide: tradizione critica della colometria e del testo

E. Calderón Dorda, 2002, Eurípides, Heracles. Ifigenia en Áulide, Madrid.

J. Diggle, 1981, Euripidis fabulae, vol. II, Oxford.

L. Dindorf, 1825, Euripidis Fabulae, vol. II, Leipzig.

J. A. Hartung, 1849, Euripides' rasender Herakles, Leipzig.

G. Hermann, 1810, Euripidis Hercules Furens, Lipsiae.

D. Kovacs, 1998, Euripides. Suppliant Women, Electra, Heracles, vol. III, Cambridge MALondon.

K. H. Lee, 1988, Euripides. Hercules, Leipzig.

M.S. Mirto, 2006, Euripide. Eracle, Milano.

G. Murray, $1913^{3}$, Euripidis Fabulae, vol. II, Oxford.

S. Musgrave, 1778, Euripidis quae extant omnia, vol. III, Oxonii.

O. Musso, 1993, Tragedie di Euripide, vol. II, Torino.

A. Nauck, $1871^{3}$, Euripidis Tragoediae, vol I, Lipsiae

F.A. Paley, $1880^{2}$, Euripides with an English Commentary, vol. III, London.

L. Parmentier \& H. Grégoire, 1923, Euripide Héraclès - Les Suppliantes - Ion, vol. III, Paris.

N. Wecklein, 1899, Euripidis Hercules, Lipsiae.

U. von Wilamowitz-Moellendorff, 1895², Euripides. Herakles, voll. I-II, Berlin.

Abbreviazioni bibliografiche

AGGS = R. Kühner \& B. Gerth, 1898-1904, Ausführliche Grammatik der griechischen Sprache, 2 vols., Hannover-Leipzig.

M. Alexiou, 2002², The Ritual Lament in Greek Tradition, Laham-Boulder-New YorkOxford.

G. Arnott, 1977, “Swan Songs"”, G\&R 24, pp. 149-153.

G. Arnott, 2007, Bird in Ancient World from A to Z, London-New York.

J. Assaël, 1996, "Le chœur de "vieux cygnes" de l'Héraclès d'Euripide”, CGITA 9, pp. 69-91.

J. Baumann, 1862, “Zu Euripides rasendem Herakles”, JPP 85, pp. 101-107.

G. Bignardi, 2013, "Osservazioni sul xúxvos in Euripide", Eikasmos 24, pp. 77-89.

G. Björck, 1950, Das "alpha impurum》 und die tragische Kunstspache, Upsala.

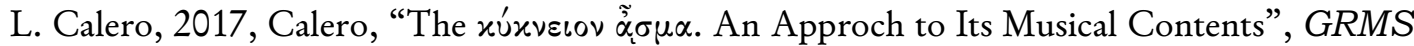
5, pp. 203-218.

E. Calderón Dorda, 2017, “Tragedia y lírica epinicial: a propósito de Eurípides, HF 673-700”, Itaca 33, pp. 9-23.

M. Cannatà, 1990, Pindarus. Threnorum fragmenta, edidit M. Cannatà Fera, Romae.

G. Comotti 2017, La musica nella cultura greca e romana, Torino.

A. M. Dale, 1983, Metrical Analyses of Tragic Choruses. vol. 3: Dochmiac-Iambic-DactylicIonic, London.

DELG = P. Chantraine, 1968-1980, Dictionnaire étymologique de la langue grecque: histoire des mots, Paris.

DGF = A. Bailly, 2020, Dictionnaire Grec-Français. Nouvelle édition revue et corrigée, sous la direction de G. Gréco, Paris.

J. Diggle, 1981, Studies on the Text of Euripides: Supplices, Electra, Heracles, Troades, Iphigenia in Tauris, Ion, Oxford.

K. Dover, 1993, Aristophanes. Frogs, Oxford.

P. Elmsley, 1813, Censura ad Herman 1810, CJ 8, 199-218.

H. Essler \& D.J. Mastronarde \& K. McNamee, 2003, “The Würzburg Scholia on Euripides' Phoenissae. A new edition of P.Würzb. 1 with Translation and Commentary", WJA 37, pp. 31-98.

$\mathrm{EDG}=\mathrm{R}$. Beekes \& L. van Beck, 2010, Etymological Dictionary of Greek, Leiden-Boston. 
Paolo Santé

La parodo dell'Eracle di Euripide: tradizione critica della colometria e del testo

M. Fantuzzi, 2007, "La mousa del lamento in Euripide, e il lamento della Musa nel Reso ascritto a Euripide", Eikasmos 18, pp. 173-199.

M.G. Fileni, 2006, Euripide. Eraclidi. I canti, Roma.

B. Gentili \& L. Lomiento, 2003, Metrica e ritmica. Storia delle forme metriche, Milano.

GGS = O. Hoffmann \& A. Debrunner, 1953-1954, Geschichte der griechischen Sprache, 2 vols., Berlin.

GP $=$ J. D. Denniston, $1954^{2}$, The Greek Particles, Oxford.

A. Grand-Clément, 2007, "Blancheur et altérité: le corps des femmes et des vieillards en Grèce ancienne", Corps 3, pp. 32-39.

B. Heath, 1762, Notae sive lectiones ad tragicorum Graecorum veterum Aeschyli Sophoclis Euripidis quae supersunt dramata deperditorumque relliquias, Oxonii.

M. Hose, 1991, Studien zum Chor bei Euripides, 2 vols., Stuttgart-Leiden.

J. Humbert, 1945, Syntaxe grecque, Paris.

J. A. Jiménez Sanchéz, 2012, “Autour des equi curules”, en Société, Économie, Administration dans le Code Théodosien, (éd. par) S. Crogiez-Pétrequin \& P. Jaillette, Villeneuve d'Ascq, pp. 481-490.

M. Kaimio, 1970, The Chorus of Greek Drama within the Light of the Person and Number Used, Helsinki.

E. Kroecker, 1938, Der Herakles des Euripides: Analyse des Dramas, Giessen.

M. Lacroix, 1967, "Notes sur quelques passages d'Euripide”, REG 80, pp. 140-147.

J. S. Lasso de la Vega, 1990, "Cincuenta notas críticas a Eurípides, Heracles furioso", $C F C(G)$ 24, pp. $19-75$.

L. Lomiento, 2008, "Appendice metrica” in Sofocle. Edipo a Colono, (a cura di) G. Avezzù \& G. Cerri \& G. Guidorizzi, Milano.

L. Lomiento, 2008a, "Il canto d'ingresso del Coro nelle 'Supplici' di Eschilo (vv. 40-175). Colometria antica e considerazioni sul rapporto tra composizione metrico-ritmica e nuclei tematici", Lexis 26, pp. 47-77.

L. Lomiento, 2019, Sofocle. Elettra, (a cura di) B. Gentili \& F. Dunn \& L. Lomiento, Milano.

L. Lomiento, 2019a, Eschilo, Supplici, (a cura di) C. Miralles \& V. Citti \& L. Lomiento, Roma.

N. Loraux, 2002, The Mourning Voice: An Essays on Greek Tragedy, transl. E.T. Rawlings, Ithaca-New York-London.

E. Medda, 2017, Eschilo, Agamennone, 3 voll., Roma.

C. Neri, 2003, "Gambe come puledre stanche (HF 119-123)", in (a cura di) O. Vox Ricerche euripidee, Lecce, pp. 65-76.

G. Pace, 2001, Euripide, Reso. I canti, Roma.

R. Palmisciano, 2017, Dialoghi per voce sola: la cultura del lamento funebre nella Grecia antica, Roma.

M. P. Pattoni 1990, "La sympatheia del coro nella parodo dei tragici greci: motivi e forme di un modello drammatico", SCO 39, pp. 33-82.

F. Perusino, 2017, Aristofane, Lisistrata. I canti, Pisa-Roma.

J. Pollard, 1977, Birds in Greek Life and Myth, London.

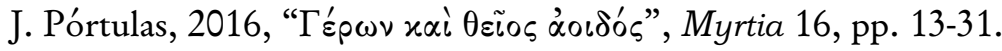

E. Rawson, 1981, "Chariot-racing in the Roman Republic", PBSR 49, pp. 1-16.

R. Rehenan, 1985, “A New Commentary on Euripides”, CPh 80, pp. 143-175.

J. J. Reiske, 1753, Ad Euripidem et Aristophanem animadversiones, Leipzig.

G. Reiter, 1962, Die griechischen Bezeichnungen der Farben Weiß, Grau und Braun, Innsbruck.

P. Santé, 2017, Euripide. Ione. I canti, Pisa-Roma.

P. Santé, 2019, “Nota critico-testuale a Eur. HF 107 119”, QUCC 117, pp. 133-138. 
Paolo Santé

La parodo dell'Eracle di Euripide: tradizione critica della colometria e del testo

M. Sanz Morales, 1988, “Notas al texto del Heracles de Eurípides”, $C F C(g)$ 21, pp. 307317.

O. Schroeder, $1928^{2}$, Euripidis Cantica, Lipsiae.

A. H. Sommerstein, 1997, Aristophanes Frogs, Warminster

A. H. Sommerstein, 1998, Aristophanes Ecclesiazusae, Warminster.

L. A. Swift, 2010, The Hidden Chorus: Echoes of Genres in Tragic Lyric, Oxford.

D. W. Thompson, 1895, A Glossay of Greek Birds, Oxford.

$\operatorname{TrGF}=$ R. Kannicht (2004), Tragicorum Graecorum Fragmenta, vol. 5, 1-2, Euripides, Göttingen.

A. Turyn, 1957, The Byzantine Manuscript Tradition of the Tragedies of Euripides, Urbana.

W. J. Verdenius, 1987, “Notes on Euripides' Heracles vv. 1-522”, Mnemosyne 40, pp. 1-17.

H. G. Viljoen, 1962, "Notes on Euripides", AClass 5, pp. 10-14.

N. Wecklein, 1874, Studien zu Euripides, Leipzig.

N. Weiss, 2017, "Noise, Music, Speech: The Representation of Lament in Greek Tragedy", AJPh 138, pp. 243-266.

M. L. West, 1973, “Critical notes on Euripides' Heracles”, Philologus 118, pp. 145-151.

M. L. West, 1982, Greek Metre, Oxford.

H. White, 2000, "Notes on the text of Euripides", Myrtia 15, pp. 51-67.

U. von Wilamowitz-Moellendorff, 1875, Analecta Euripidea, Berolini.

U. von Wilamowitz-Moellendorff, 1921, Griechische Verskunst, Berlin.

C. Willink, 2004, "Critical notes on the cantica of Euripides' Heracles", Philologus 148, pp. 197-221.

G. Zuntz, 1965, An Inquiry into the Transmission of the Plays of Euripides, London. 